\title{
Hermitian symmetric spaces and Kähler rigidity
}

\section{Journal Article}

\section{Author(s):}

Burger, Marc; lozzi, Alessandra; Wienhard, Anna

Publication date:

2007

Permanent link:

https://doi.org/10.3929/ethz-b-000006775

Rights / license:

In Copyright - Non-Commercial Use Permitted

Originally published in:

Transformation groups 12(1), https://doi.org/10.1007/s00031-005-1135-0 


\title{
HERMITIAN SYMMETRIC SPACES AND KÄHLER RIGIDITY
}

MARC BURGER

FIM, ETH Zentrum

Rämistrasse 101

CH-8092 Zürich, Switzerland

burger@math.ethz.ch
ALESSANDRA IOZZI*

Institut für Mathematik

Universität Basel

Rheinsprung 21

CH-4051 Basel, Switzerland

Départment de Mathématiques

Université de Strasbourg

7, rue René Descartes

F-67084 Strasbourg Cedex, France

iozzi@math.ethz.ch

\author{
ANNA WIENHARD \\ School of Mathematics \\ Institute for Advanced Study \\ 1 Einstein Drive \\ Princeton, NJ 08540, USA \\ Department of Mathematics, \\ University of Chicago, \\ 5734 S. University Ave., \\ Chicago, IL 60637, USA \\ wienhard@math.uchicago.edu
}

\begin{abstract}
We characterize irreducible Hermitian symmetric spaces which are not of tube type, both in terms of the topology of the space of triples of pairwise transverse points in the Shilov boundary, and of two invariants which we introduce, the Hermitian triple product and its complexification. We apply these results and the techniques introduced in [6] to characterize conjugacy classes of Zariski dense representations of a locally compact group into the connected component $G$ of the isometry group of an irreducible Hermitian symmetric space which is not of tube type, in terms of the pullback of the bounded Kähler class via the representation. We conclude also that if the second bounded cohomology of a finitely generated group $\Gamma$ is finite dimensional, then there are only finitely many conjugacy classes of representations of $\Gamma$ into $G$ with Zariski dense image. This generalizes results of [6].
\end{abstract}

DOI: $10.1007 / \mathrm{s} 00031-005-1135-0$.

${ }^{*}$ A. I. and A. W. were partially supported by FNS grant PP002-102765. A. W. was partially supported by the National Science Foundation under agreement No. DMS-0111298.

Received December 4, 2005. Accepted May 29, 2006. Published online February 15, 2007. 


\section{Introduction}

A symmetric space $\mathcal{X}$ is Hermitian if it carries a complex structure which is $\operatorname{Isom}(\mathcal{X})^{\circ}$ invariant and hence, together with the Riemannian metric, defines a closed $\operatorname{Isom}(\mathcal{X})^{\circ}$ invariant differential two-form on $\mathcal{X}$, which thus turns $\mathcal{X}$ into a Kähler manifold. The Kähler form gives rise to invariants for isometric actions of finitely generated groups $\Gamma$ on $\mathcal{X}$ itself and on geometric objects related to $\mathcal{X}$.

Rigidity results involving these invariants were proven first by Ghys [21] and Matsumoto [31] (see also [26]) for actions of $\Gamma$ by orientation preserving homeomorphisms on $S^{1}$ by interpreting the orientation cocycle on $S^{1}$ as a bounded cohomology class. The orientation cocycle is in turn, up to a multiple, the extension to the boundary of the "area function" on geodesic triangles in the Poincare disk which associates to an ordered triple the area of the geodesic triangle determined by it.

Likewise, when $\mathcal{X}$ is the complex hyperbolic space $\mathcal{H}_{\mathbb{C}}^{n}$ the angular invariant defined by Cartan in [12] is a real valued bounded function on ordered triples in $\partial \mathcal{H}_{\mathbb{C}}^{n}$ and is, up to a constant, the bounded cocycle defined by integrating the Kähler form on $\mathcal{H}_{\mathbb{C}}^{n}$ over geodesic triangles (see [22] for definition and background). The interpretation of Cartan's invariant as a bounded cohomology class has been applied by Toledo [35] to study representations of surface groups into the isometry group of $\mathcal{H}_{\mathbb{C}}^{n}$ and recently by the first two authors [26], [2], [4], [3] to reprove and extend the results of Goldman and Millson [23] on deformation rigidity of complex hyperbolic lattices (see also [30] for an approach by harmonic maps).

More generally, let $\mathcal{X}$ be a Hermitian symmetric space of noncompact type and let $G=\operatorname{Isom}(\mathcal{X})^{\circ}$ be the connected component of its group of isometries. Then $\mathcal{X}$ is biholomorphic to a bounded domain $\mathcal{D}$ in a complex vector space on which $G$ acts by birational transformations defined on and preserving $\overline{\mathcal{D}}$. The Bergmann metric on $\mathcal{D}$ is Kähler of course and denoting by $\omega_{\text {Berg }}$ its Bergmann-Kähler form and by $\Delta(x, y, z) \subset \mathcal{D}$ a smooth triangle with geodesic sides, the function on $G^{3}$,

$$
c_{\omega_{\text {Berg }}}\left(g_{1}, g_{2}, g_{3}\right):=\frac{1}{2 \pi} \int_{\Delta\left(g_{1} 0, g_{2} 0, g_{3} 0\right)} \omega_{\text {Berg }}, \quad 0 \in \mathcal{D},
$$

defines a $G$-invariant continuous (homogeneous) two-cocycle which is bounded [19] and thus defines both a continuous class $\kappa_{G, \mathrm{~B}} \in \mathrm{H}_{\mathrm{c}}^{2}(G, \mathbb{R})$ and a bounded continuous class $\kappa_{G, \mathrm{~B}}^{\mathrm{b}} \in \mathrm{H}_{\mathrm{cb}}^{2}(G, \mathbb{R})$, which correspond to each other via the comparison map $\mathrm{H}_{\mathrm{cb}}^{2}(G, \mathbb{R}) \rightarrow$ $\mathrm{H}_{\mathrm{c}}^{2}(G, \mathbb{R})$ and are called, respectively, the Kähler class and the bounded Kähler class. Now let $H$ be a locally compact group and $\rho: H \rightarrow G$ a continuous representation. The invariant

$$
\rho^{*}\left(\kappa_{G, \mathrm{~B}}^{\mathrm{b}}\right) \in \mathrm{H}_{\mathrm{cb}}^{2}(H, \mathbb{R})
$$

is the bounded Kähler class of $\rho$ and the question to which extent this invariant determines $\rho$ has been addressed by Ghys for actions by orientation preserving homeomorphisms of the circle in [21] and by the first two authors for $G=\mathrm{SU}(p, q)$ in [6], where complete results were obtained. For instance, if $p \neq q$ and the image is Zariski dense, then $\rho$ is determined (up to conjugation) by its bounded Kähler class.

One of the aims of this paper is to extend this type of results to more general (irreducible) Hermitian symmetric spaces. Along the way we will have to face the issue 
of the proper generalization of the necessary hypothesis $p \neq q$. This will bring in the notion of tube type domain and lead us to establish a new characterization of domains which are not of tube type, topics to which we now turn in more detail.

The space $\mathcal{X}$ (or the domain $\mathcal{D}$ ) is of tube type if it is biholomorphic to a domain of the form

$$
V \oplus i \Omega \subset V \oplus i V
$$

where $V$ is a real vector space and $\Omega \subset V$ is a convex open cone. A typical example is the symmetric space associated to $\mathrm{SU}(p, q)$, which is of tube type if and only if $p=q$, in which case it is biholomorphic to

$$
\operatorname{Herm}_{p}(\mathbb{C})+i \operatorname{Herm}_{p}^{+}(\mathbb{C}),
$$

where $\operatorname{Herm}_{p}(\mathbb{C})$ is the vector space of Hermitian matrices and $\operatorname{Herm}_{p}^{+}(\mathbb{C})$ is the open cone of positive definite ones.

A basic object in this context is the Shilov boundary $\check{S} \subset \partial \mathcal{D}$ of the bounded domain realization $\mathcal{D}$ of the symmetric space; its topological properties reflect analytic-geometric properties of $\mathcal{D}$, an example being the classical characterization of tube type domains as those for which $\pi_{1}(\check{S})$ is infinite. One of our characterizations below of domains which are not of tube type will give one more instance of this interplay. In order to state this we will need to introduce two more objects, namely, the Hermitian triple product and its "complexification".

The Bergmann kernel $k_{\mathcal{D}}: \mathcal{D} \times \mathcal{D} \rightarrow \mathbb{C}^{\times}$extends continuously to the subset $\check{S}^{(2)} \subset$ $\check{S} \times \check{S}$ consisting of pairs of transverse points, that is, to the unique open $G$-orbit in $\breve{S} \times \check{S} \cong G / Q \times G / Q$, where $Q$ is a specific maximal parabolic subgroup; on the subset $\check{S}^{(3)} \subset \breve{S}^{3}$ consisting of triples of pairwise transverse points, we define the Hermitian triple product

$$
\langle\langle\cdot, \cdot, \cdot\rangle\rangle: \check{S}^{(3)} \longrightarrow \mathbb{R}^{\times} \backslash \mathbb{C}^{\times}, \quad(x, y, z) \mapsto k_{\mathcal{D}}(x, y) k_{\mathcal{D}}(y, z) k_{\mathcal{D}}(z, x) \bmod \mathbb{R}^{\times},
$$

which is a $G$-invariant multiplicative cocycle on $\check{S}^{(3)}$. If now $\mathbf{G}$ is the connected adjoint $\mathbb{R}$-group associated to the complexification of the Lie algebra of $G$ - so that $G=\mathbf{G}(\mathbb{R})^{\circ}$ - and $\mathbf{Q}<\mathbf{G}$ is the parabolic subgroup with $\mathbf{Q}(\mathbb{R})=Q$, then we have a natural identification

$$
\imath: \check{S} \longrightarrow \mathbf{G} / \mathbf{Q}(\mathbb{R})
$$

of the Shilov boundary $\check{S}$ with the real points of the complex projective variety $\mathbf{G} / \mathbf{Q}$. We define in Section 2.4 a $\mathbf{G}$-invariant rational function $\langle\langle\cdot, \cdot, \cdot\rangle\rangle_{\mathbb{C}}$ on $(\mathbf{G} / \mathbf{Q})^{3}$ which extends the Hermitian triple product via the identification $\imath$ and is hence called the complex Hermitian triple product.

Theorem 1. Let $\mathcal{X}$ be an irreducible Hermitian symmetric space. The following are equivalent:

(1) $\mathcal{X}$ is not of tube type;

(2) the set $\check{S}^{(3)}$ of triples of pairwise transverse points in the Shilov boundary is connected;

(3) the Hermitian triple product $\langle\langle\cdot, \cdot, \cdot\rangle\rangle: \check{S}^{(3)} \rightarrow \mathbb{R}^{\times} \backslash \mathbb{C}^{\times}$is not constant.

(4) the complex Hermitian triple product $\langle\langle\cdot, \cdot, \cdot\rangle\rangle_{\mathbb{C}}$ on $(\mathbf{G} / \mathbf{Q})^{3}$ is not constant. 
Remark 2. (1) For the convenience of the reader we recall that the classification of all irreducible Hermitian symmetric spaces, according as to whether or not they are of tube type, is as follows:

\begin{tabular}{c|c} 
tube type & nontube type \\
\hline \hline $\mathrm{SU}(p, p)$ & $\begin{array}{c}\mathrm{SU}(p, q) \\
p<q\end{array}$ \\
\hline $\mathrm{Sp}(2 n, \mathbb{R})$ & \\
\hline $\begin{array}{c}\mathrm{SO}^{*}(2 n) \\
n \text { even }\end{array}$ & $\begin{array}{c}\mathrm{SO}^{*}(2 n) \\
n \text { odd }\end{array}$ \\
\hline $\mathrm{SO}(2, n)$ & \\
\hline $\mathrm{E}_{7}(-25)$ & $\mathrm{E}_{6}(-14)$ \\
\hline
\end{tabular}

where $\mathrm{E}_{7}(-25)$ and $\mathrm{E}_{6}(-14)$ correspond to the exceptional Hermitian symmetric spaces of complex dimension 27 and 16, respectively.

(2) Invariants related to the Hermitian triple product have been introduced and studied in various contexts and we refer the interested reader to [6], [13], [16], [17], [15], [14].

The above theorem is an essential ingredient in our investigation of the geometric properties of maximal representations of compact surface groups [9], [8], [7]. It is however also one of the essential building blocks in the following general theorem characterizing representations in terms of their bounded Kähler class.

Theorem 3. Let $H$ be a locally compact second countable group, $\mathcal{X}$ an irreducible Hermitian symmetric space which is not of tube type and $\rho: H \rightarrow G:=\operatorname{Isom}(\mathcal{X})^{\circ}$ a continuous homomorphism with Zariski dense image. Then the bounded Kähler class $\rho^{*}\left(\kappa_{G, \mathrm{~B}}^{\mathrm{b}}\right)$ is nonzero and determines $\rho$ up to conjugation.

The hypothesis that $\mathcal{X}$ is not of tube type is necessary. Indeed, let $\Gamma<\mathrm{PU}(1,1)$ be a torsion free cocompact lattice, and $\rho: \Gamma \rightarrow G$ a maximal representation (for results and notions pertinent to the theory of maximal representations we refer the reader to [9], [8], [7]). Then:

(1) $\rho^{*}\left(\kappa_{G, \mathrm{~B}}^{\mathrm{b}}\right)=\lambda_{\mathcal{X}} \kappa_{\mathrm{PU}(1,1), \mathrm{B}}^{\mathrm{b}}$, where $\lambda_{\mathcal{X}}$ is some constant depending only on $\mathcal{X}$;

(2) if $\mathcal{X}$ is of tube type, there exist one-parameter families of maximal representations which have Zariski dense image in $G$ and are inequivalent modulo conjugation.

Theorem 3 will follow in fact from the next more general result. Recall that we assume that all the Hermitian symmetric spaces are equipped with their Bergmann metric and let us convene that if $\rho_{i}: H \rightarrow G_{i}:=\operatorname{Isom}\left(\mathcal{X}_{i}\right)^{\circ}$ for $i=1,2$ are continuous representations, we say that $\left(\rho_{1}, \mathcal{X}_{1}\right)$ and $\left(\rho_{2}, \mathcal{X}_{2}\right)$ are equivalent if there is an isometry $T: \mathcal{X}_{1} \rightarrow \mathcal{X}_{2}$ which intertwines the two actions.

Theorem 4. Let $H$ be a locally compact second countable group and let $\left(\rho_{j}, \mathcal{X}_{j}\right), j=$ $1, \ldots, n$, be continuous isometric actions of $H$ on irreducible Hermitian symmetric spaces. Assume that: 
(1) for every $1 \leqslant j \leqslant n, \mathcal{X}_{j}$ is not of tube type;

(2) the actions are pairwise inequivalent; and

(3) $\rho_{j}(H)$ is Zariski dense in $G_{j}:=\operatorname{Isom}\left(\mathcal{X}_{j}\right)^{\circ}$ for $1 \leqslant j \leqslant n$.

Then the subset

$$
\left\{\rho_{j}^{*}\left(\kappa_{G_{j}, \mathrm{~B}}^{\mathrm{b}}\right) \mid 1 \leqslant j \leqslant n\right\} \subset \mathrm{H}_{\mathrm{cb}}^{2}(H, \mathbb{R})
$$

is linearly independent over $\mathbb{Z}$.

Let $\Gamma$ be a finitely generated group and let $\operatorname{Rep}_{Z D}(\Gamma, G)$ be the set of representations of $\Gamma$ into $G$ with Zariski dense image, modulo $G$-conjugation. This quotient has a natural structure of real semialgebraic set and, according to Theorem 3 , if $\mathcal{X}$ is not of tube type the continuous map

$$
\operatorname{Rep}_{Z D}(\Gamma, G) \longrightarrow \mathrm{H}_{\mathrm{b}}^{2}(\Gamma, \mathbb{R}), \quad[\rho] \mapsto \rho^{*}\left(\kappa_{G, \mathrm{~B}}^{\mathrm{b}}\right),
$$

is injective. This, together with Theorem 4, will imply the following

Corollary 5. Let $\Gamma$ be a finitely generated group and $G:=\operatorname{Isom}(\mathcal{X})^{\circ}$, where $\mathcal{X}$ is an irreducible Hermitian symmetric space. Assume that:

(1) $\mathcal{X}$ is not of tube type; and

(2) $\mathrm{H}_{\mathrm{b}}^{2}(\Gamma, \mathbb{R})$ is finite dimensional.

Then there are, up to $G$-conjugation, only finitely many representations $\Gamma \rightarrow G$ with Zariski dense image.

The Kähler class $\kappa_{G, \mathrm{~B}} \in \mathrm{H}_{\mathrm{c}}^{2}(G, \mathbb{R})$ associated to the Bergmann metric comes from an integral class, namely, is in the image of the $\operatorname{map~}_{\mathrm{c}}^{2}(G, \mathbb{Z}) \rightarrow \mathrm{H}_{\mathrm{c}}^{2}(G, \mathbb{R})$ (see Section 4). Using this integral representative we also obtain a generalization of the finiteness result of $[6$, Cor. 1.6]:

Corollary 6. Assume that $\Gamma$ is finitely generated and that the comparison $m a p \mathrm{H}_{\mathrm{b}}^{2}(\Gamma, \mathbb{R})$ $\rightarrow \mathrm{H}^{2}(\Gamma, \mathbb{R})$ is injective. Let $\mathcal{X}$ be an irreducible Hermitian symmetric space which is not of tube type and let $G:=\operatorname{Isom}(\mathcal{X})^{\circ}$. Then the number of conjugacy classes of representations of $\Gamma$ into $G$ with Zariski dense image is bounded by $\operatorname{dim}_{\mathbb{R}} \mathrm{H}^{2}(\Gamma, \mathbb{R})$.

\section{Outline of the paper}

In Section 2, after having recalled (Sections 2.1 and 2.2) basic facts about the Borel and the Harish-Chandra realizations of a Hermitian symmetric space, we give (Section 2.3) a model for the complexification of the Shilov boundary; then we introduce and study (Section 2.4) the Hermitian triple product and its complexification, and establish the relation between these invariants in Corollary 2.17.

In Section 3 we prove Theorem 1: we study the decomposition of the Shilov boundary into Bruhat cells (Section 3.1) and deduce our topological characterizations of domains not of tube type in terms of the connectedness of the set of triples of pairwise transverse points. Then (Section 3.2), using the work of Clerc and Ørsted, we consider the Bergmann cocycle on the Shilov boundary, obtained by continuous extension of the Kähler integral on geodesic triangles; the properties of this cocycle together with its relation to the Hermitian triple product allow us to finish the proof of Theorem 1. 
In Section 4 we collect the ingredients in order to prove the remaining results, using and simplifying the strategy in [6]. First (Section 4.1) we introduce the Kähler class and show that it comes from an "integral continuous" class on $G$. In Sections 4.2 and 4.3 we apply basic techniques from the theory of bounded continuous cohomology as developed in [11], [32], [5]. In Section 4.2 we use the Bergmann cocycle on the Shilov boundary to give a natural realization of the bounded Kähler class, and in Section 4.3 we establish in general (as in [6] for $G=\mathrm{SU}(p, q)$ ) the formula for the bounded Kähler class of a representation using boundary maps. In Section 4.4 we recall a classical result on the existence of boundary maps.

In Section 5 we finally prove Theorems 3 and 4 and Corollaries 5 and 6 .

\section{Hermitian triple product}

We recall the necessary background material about the structure of Hermitian symmetric spaces. We follow mostly the paper by A. Korányi [28]; for proofs the reader is referred to this paper and the references therein.

Let $\mathcal{X}$ be a symmetric space of noncompact type and $G=\operatorname{Isom}(\mathcal{X})^{\circ}$ the connected component of the identity of the group of isometries of $\mathcal{X}$. Recall that $r_{\mathcal{X}}$, the rank of $\mathcal{X}$, is the maximal dimension of an isometrically and totally geodesic embedded Euclidean subspace in $\mathcal{X}$.

Definition 2.1. A symmetric space $\mathcal{X}$ is Hermitian if it admits a $G$-invariant complex structure. We call a connected semisimple real algebraic group of Hermitian type if its associated symmetric space is Hermitian.

Example 2.2. A typical example, which will serve as an illustration throughout this paper, is the symmetric space associated to $\mathrm{SU}(p, q)$. Let $\mathbb{C}^{n}$ be endowed with a nondegenerate Hermitian form $\langle\cdot, \cdot\rangle$ of signature $(p, q)$ given by

$$
\langle z, w\rangle:=\sum_{j=1}^{p} z_{j} \overline{w_{j}}-\sum_{j=p+1}^{n} z_{j} \overline{w_{j}}
$$

with respect to the standard basis $\left(e_{1}, \ldots, e_{n}\right)$. Up to a change of sign we may assume that $p \leqslant q$. The Lie group

$$
G_{p, q}:=\mathrm{SU}(p, q)=\{g \in \mathrm{SL}(n, \mathbb{C}) \mid\langle g \cdot, g \cdot\rangle=\langle\cdot, \cdot\rangle\}
$$

is of Hermitian type with associated symmetric space

$$
\mathcal{X}_{p, q}=\left\{L \in \operatorname{Gr}_{p}\left(\mathbb{C}^{n}\right)|\langle\cdot, \cdot\rangle|_{L} \text { is positive definite }\right\},
$$

which is of rank $p$. Choosing $L_{+}=\left\langle e_{1}, \ldots, e_{p}\right\rangle$ as a base point, we have the identification $\mathcal{X}_{p, q}=G_{p, q} / K_{p, q}$ where $K_{p, q}=\operatorname{Stab}_{G_{p, q}}\left(L_{+}\right)=\mathrm{S}(\mathrm{U}(p) \times \mathrm{U}(q))$ is a maximal compact subgroup. The embedding of $\mathcal{X}_{p, q}$ as an open subspace in the complex Grassmannian $\operatorname{Gr}_{p}\left(\mathbb{C}^{n}\right)$ is the Borel embedding, which naturally induces a $G_{p, q}$-invariant complex structure on $\mathcal{X}_{p, q}$; the closure of the image

$$
\overline{\mathcal{X}}_{p, q}=\left\{L \in \operatorname{Gr}_{p}\left(\mathbb{C}^{n}\right)|\langle\cdot, \cdot\rangle|_{L} \text { is semipositive definite }\right\}
$$

is a compactification of $\mathcal{X}_{p, q}$. 


\subsection{The Borel embedding and the Harish-Chandra embedding}

Let $\mathcal{X}$ be a Hermitian symmetric space of noncompact type with a fixed $G$-invariant complex structure. The stabilizer $K:=\operatorname{Stab}_{G}\left(x_{0}\right)$ of a given point $x_{0} \in \mathcal{X}$ is a maximal compact subgroup of $G$ and the Lie algebra $\mathfrak{g}$ of $G$ decomposes as a direct sum $\mathfrak{g}=\mathfrak{k} \oplus \mathfrak{p}$, where $\mathfrak{k}$ is the Lie algebra of $K$, orthogonal with respect to the Killing form $\mathfrak{B}: \mathfrak{g} \times \mathfrak{g} \rightarrow \mathbb{R}$.

The fact that $\mathcal{X}$ is Hermitian implies that there exists a unique element $Z_{0}$ in the center $\mathcal{Z}(\mathfrak{k})$ of $\mathfrak{k}$ such that $\left.\operatorname{ad}\left(Z_{0}\right)\right|_{\mathfrak{p}}$ induces the complex structure under the identification $\mathfrak{p} \cong T_{x_{0}} \mathcal{X} ;$ if, in addition, $\mathcal{X}$ is irreducible, then $Z_{0}$ spans $\mathcal{Z}(\mathfrak{k})$.

Denoting by $\mathfrak{l}_{\mathbb{C}}$ the complexification $\mathfrak{l} \otimes \mathbb{C}$ of a real Lie algebra $\mathfrak{l}, \mathfrak{g}_{\mathbb{C}}$ splits under the action of the endomorphism $\operatorname{ad}\left(Z_{0}\right)$ into eigenspaces,

$$
\mathfrak{g}_{\mathbb{C}}=\mathfrak{k}_{\mathbb{C}} \oplus \mathfrak{p}_{+} \oplus \mathfrak{p}_{-},
$$

where $\mathfrak{p}_{ \pm}$are the $( \pm i)$-eigenspaces and $\mathfrak{k}_{\mathbb{C}}$ is the zero-eigenspace. In particular, if $\epsilon \in$ $\{+,-\},\left[\mathfrak{p}_{\epsilon}, \mathfrak{p}_{\epsilon}\right]=0$ and $\left[\mathfrak{k}_{\mathbb{C}}, \mathfrak{p}_{\epsilon}\right] \subset \mathfrak{p}_{\epsilon}$.

The group $\mathbf{G}=\operatorname{Aut}\left(\mathfrak{g}_{\mathbb{C}}\right)^{\circ}$ is a connected linear algebraic group defined over $\mathbb{R}$ whose Lie algebra is identified with $\mathfrak{g}_{\mathbb{C}}$ via the adjoint representation ad, and its set of real points $\mathbf{G}(\mathbb{R})^{\circ}=\operatorname{Aut}(\mathfrak{g})^{\circ}$ is identified with $G$ via the adjoint representation Ad. Denote by $P_{+}, P_{-}, K_{\mathbb{C}}$ the analytic subgroups of $\mathbf{G}$ with Lie algebras $\mathfrak{p}_{+}, \mathfrak{p}_{-}, \mathfrak{k}_{\mathbb{C}}$, respectively. Then $P_{+}, P_{-}, K_{\mathbb{C}}$ are algebraic subgroups and $K_{\mathbb{C}}$ normalizes $P_{+}$and $P_{-}$. The semidirect products $K_{\mathbb{C}} P_{+}$and $K_{\mathbb{C}} P_{-}$are opposite parabolic subgroups of $\mathbf{G}$ with unipotent radicals $P_{+}$and $P_{-}$, respectively. Hence the product map induces an isomorphism of varieties of $P_{\epsilon} \times K_{\mathbb{C}} P_{-\epsilon}$ onto the Zariski open and dense subset $\mathcal{O}_{\epsilon}:=P_{\epsilon} K_{\mathbb{C}} P_{-\epsilon}$ of $\mathbf{G}$ for $\epsilon \in\{+,-\}$ [1, Prop. 14.21]. In particular $P_{\epsilon} \cap K_{\mathbb{C}} P_{-\epsilon}=\{e\}$, and the maps

$$
\begin{aligned}
\pi_{\epsilon}^{\epsilon}: & \mathcal{O}_{\epsilon} \longrightarrow P_{\epsilon}, \\
\pi_{0}^{\epsilon}: \mathcal{O}_{\epsilon} & \longrightarrow K_{\mathbb{C}}, \\
\pi_{-\epsilon}^{\epsilon}: & \mathcal{O}_{\epsilon} \longrightarrow P_{-\epsilon},
\end{aligned}
$$

defined by the uniqueness of the decompositions

$$
g:=\pi_{\epsilon}^{\epsilon}(g) \pi_{0}^{\epsilon}(g) \pi_{-\epsilon}^{\epsilon}(g)
$$

are regular.

The homogeneous spaces

$$
M_{\epsilon}:=\mathbf{G} / K_{\mathbb{C}} P_{-\epsilon}
$$

are complex projective varieties and, since $K=G \cap K_{\mathbb{C}} P_{\epsilon}$, one obtains the Borel embeddings $b_{\epsilon}$ of the symmetric space $\mathcal{X}$ into the projective varieties $M_{\epsilon}$ : these are holomorphic embeddings defined by

$$
b_{\epsilon}: \mathcal{X}=G / K \longrightarrow M_{\epsilon}, \quad g K \mapsto g x_{\epsilon}
$$

where $x_{\epsilon}:=K_{\mathbb{C}} P_{\epsilon}$ for $\epsilon \in\{+,-\}$.

If exp denotes the exponential map on $\mathbf{G}$, we have 
Lemma 2.3. The map

$$
\xi_{\epsilon}: \mathfrak{p}_{\epsilon} \longrightarrow M_{\epsilon}, \quad z \mapsto(\exp z) x_{\epsilon}
$$

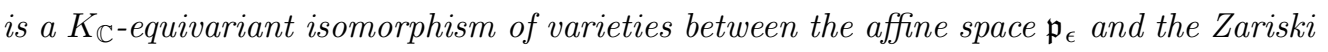
open subset $U_{\epsilon}:=P_{\epsilon} x_{\epsilon} \subset M_{\epsilon}$.

Proof. Since $P_{\epsilon}$ is Abelian, the exponential map exp $: \mathfrak{p}_{\epsilon} \rightarrow P_{\epsilon}$ is an isomorphism of varieties. The map $\xi_{\epsilon}$ is the composition of the isomorphism exp with the isomorphism of varieties $P_{\epsilon} \rightarrow U_{\epsilon}$ induced by the orbit map $P_{\epsilon} \rightarrow M_{\epsilon}$ [1, Prop. 14.12].

Since we have the inclusion $G \subset P_{\epsilon} K_{\mathbb{C}} P_{-\epsilon}$, the image of the Borel embedding $b_{\epsilon}$ is contained in $U_{\epsilon}$, and hence, by Lemma 2.3 , we may compose

$$
\mathcal{X}=G / K \stackrel{b_{\epsilon}}{\longrightarrow} U_{\epsilon} \stackrel{\xi_{\epsilon}^{-1}}{\longrightarrow} \mathfrak{p}_{\epsilon}
$$

to obtain the Harish-Chandra embeddings. The image

$$
\mathcal{D}_{\epsilon}:=\xi_{\epsilon}^{-1} \circ b_{\epsilon}(\mathcal{X}) \subset \mathfrak{p}_{\epsilon}
$$

is a bounded symmetric domain in the complex vector space $\mathfrak{p}_{\epsilon}$, called the HarishChandra realization of $\mathcal{X}$. A more precise description of $\mathcal{D}_{\epsilon}$ requires the notion of strongly orthogonal roots which we recall in the following section.

Example 2.4. The Lie algebra $\mathfrak{g}_{p, q}$ of $G_{p, q}$ is given by

$$
\mathfrak{g}_{p, q}=\mathfrak{s u}(p, q):=\{X \in \mathfrak{s l}(n, \mathbb{C}) \mid\langle X \cdot, \cdot\rangle+\langle\cdot, X \cdot\rangle=0\}
$$

writing $X=\left(\begin{array}{ll}A & B \\ C & D\end{array}\right) \in \mathfrak{s u}(p, q)$ with $A \in \operatorname{Mat}(p, p, \mathbb{C}), B \in \operatorname{Mat}(p, q, \mathbb{C}), C \in$ $\operatorname{Mat}(q, p, \mathbb{C})$, and $D \in \operatorname{Mat}(q, q, \mathbb{C})$, we have

$$
A^{*}=-A, \quad D^{*}=-D, \quad B^{*}=C, \operatorname{tr}(X)=0 .
$$

The central element in $\mathfrak{k}$ inducing the complex structure is

$$
Z_{0}=\left(\begin{array}{cc}
\frac{i q}{p+q} \operatorname{Id}_{p} & 0 \\
0 & -\frac{i p}{p+q} \operatorname{Id}_{q}
\end{array}\right)
$$

and gives rise to the following decomposition of the complexification $\mathfrak{g}_{\mathbb{C}}=\mathfrak{s l}(n, \mathbb{C})$,

$$
\begin{aligned}
& \mathfrak{k}_{\mathbb{C}}=\left\{X \in \mathfrak{s l}(n, \mathbb{C}) \mid X=\left(\begin{array}{ll}
A & 0 \\
0 & D
\end{array}\right)\right\}, \\
& \mathfrak{p}_{+}=\left\{X \in \mathfrak{s l}(n, \mathbb{C}) \mid X=\left(\begin{array}{ll}
0 & B \\
0 & 0
\end{array}\right)\right\} \simeq \operatorname{Mat}(p, q, \mathbb{C}), \\
& \mathfrak{p}_{-}=\left\{X \in \mathfrak{s l}(n, \mathbb{C}) \mid X=\left(\begin{array}{ll}
0 & 0 \\
C & 0
\end{array}\right)\right\} \simeq \operatorname{Mat}(q, p, \mathbb{C}) .
\end{aligned}
$$


The Harish-Chandra realization (see Section 2.2) can be described geometrically by fixing the base point $L_{+}$with orthogonal complement $L_{-}$. Since the orthogonal projection $\left.\operatorname{pr}_{L_{+}}\right|_{L}: L \rightarrow L_{+}$is an isomorphism, every $L \in \overline{\mathcal{X}_{p, q}}$ can be realized as graph of a unique linear map

$$
Z_{L}:=\left.\operatorname{pr}\right|_{L_{-}} \circ\left(\left.\operatorname{pr}_{L_{+}}\right|_{L}\right)^{-1}: L_{+} \rightarrow L_{-}
$$

and the map

$$
\overline{\mathcal{X}}_{p, q} \longrightarrow \operatorname{Mat}(q, p, \mathbb{C}), \quad L \mapsto Z_{L},
$$

gives an identification of $\mathcal{X}_{p, q}$ with the bounded symmetric domain

$$
\mathcal{D}_{p, q}=\left\{Z \in \operatorname{Mat}(q, p, \mathbb{C}) \mid \operatorname{Id}_{p}-Z^{*} Z \text { is positive definite }\right\} \subset \mathfrak{p}_{-}
$$

and of $\overline{\mathcal{X}}_{p, q}$ with

$$
\overline{\mathcal{D}}_{p, q}=\left\{Z \in \operatorname{Mat}(q, p, \mathbb{C}) \mid \operatorname{Id}_{p}-Z^{*} Z \text { is semipositive definite }\right\} \subset \mathfrak{p}_{-} .
$$

\subsection{The Harish-Chandra realizations as bounded symmetric domains}

Let $\mathfrak{h} \subset \mathfrak{k}$ be a maximal Abelian subalgebra. Then $\mathcal{Z}(\mathfrak{k}) \subset \mathfrak{h}$ so that the complexification $\mathfrak{h}_{\mathbb{C}} \subset \mathfrak{g}_{\mathbb{C}}$ is also maximal and can be checked to be a Cartan subalgebra in $\mathfrak{g}_{\mathbb{C}}$. Let $\Psi=\Psi\left(\mathfrak{g}_{\mathbb{C}}, \mathfrak{h}_{\mathbb{C}}\right)$ be the set of roots of $\mathfrak{h}_{\mathbb{C}}$ in $\mathfrak{g}_{\mathbb{C}}$ and let

$$
\mathfrak{g}_{\mathbb{C}}=\mathfrak{h}_{\mathbb{C}} \oplus \bigoplus_{\alpha \in \Psi} \mathfrak{g}_{\alpha}
$$

be the corresponding root space decomposition. Define

$$
\Psi^{\mathfrak{k}_{\mathbb{C}}}:=\left\{\alpha \in \Psi \mid \mathfrak{g}_{\alpha} \subset \mathfrak{k}_{\mathbb{C}}\right\}
$$

the set of compact roots and

$$
\Psi^{\mathfrak{p}_{\epsilon}}:=\left\{\alpha \in \Psi \mid \mathfrak{g}_{\alpha} \subset \mathfrak{p}_{\epsilon}\right\} .
$$

Choose an ordering $\Psi=\Psi_{+} \sqcup \Psi_{-}$such that $\Psi^{\mathfrak{p}_{\epsilon}} \subset \Psi_{\epsilon}$ and refer to $\Psi^{\mathfrak{p}_{+}}$(respectively, to $\Psi^{\mathfrak{p}_{-}}$) as the set of positive (respectively, negative) noncompact roots.

To each root $\alpha \in \Psi$ associate a three-dimensional simple subalgebra

$$
\mathfrak{g}[\alpha]=\mathbb{C} H_{\alpha} \oplus \mathbb{C} E_{\alpha} \oplus \mathbb{C} E_{-\alpha} \subset \mathfrak{g}_{\mathbb{C}}
$$

as follows: $H_{\alpha} \in \mathfrak{h}_{\mathbb{C}}$ is the unique element determined by

$$
\alpha(H)=2 \frac{\mathfrak{B}\left(H, H_{\alpha}\right)}{\mathfrak{B}\left(H_{\alpha}, H_{\alpha}\right)} \quad \text { for all } H \in \mathfrak{h}_{\mathbb{C}}
$$

where (by a slight abuse of notation) $\mathfrak{B}$ is the Killing form of $\mathfrak{g}_{\mathbb{C}}$ and, since $\mathfrak{g}_{\alpha}$ is one dimensional, $E_{\alpha}$ and $E_{-\alpha}$ are the unique elements defined by the relations

$$
\left[E_{\alpha}, E_{-\alpha}\right]=H_{\alpha} \text { and } \tau\left(E_{\alpha}\right)=-E_{-\alpha},
$$

where $\tau$ is the complex conjugation of $\mathfrak{g}_{\mathbb{C}}$ with respect to the compact real form $\mathfrak{g}_{U}=$ $\mathfrak{k} \oplus i \mathfrak{p}$. Then $H_{\alpha} \in i \mathfrak{h}$,

$$
\mathfrak{p}_{\epsilon}=\sum_{\alpha \in \Psi^{\mathfrak{p}_{\epsilon}}} \mathbb{C} E_{\alpha},
$$

and the elements $X_{\alpha}=E_{\alpha}+E_{-\alpha}$ together with $Y_{\alpha}=i\left(E_{\alpha}-E_{-\alpha}\right)$, for $\alpha \in \Psi^{\mathfrak{p}+}$, form a real basis of $\mathfrak{p}$. 
Example 2.5. When $\mathfrak{g}=\mathfrak{s u}(p, q), \mathfrak{g}_{\mathbb{C}}=\mathfrak{s l}(n, \mathbb{C})$, and the Cartan subgroup can be chosen to be

$$
\mathfrak{h}_{\mathbb{C}}=\left\{H=\operatorname{diag}\left(h_{1}, \ldots, h_{n}\right) \mid \sum_{j=1}^{n} h_{j}=0\right\} .
$$

The roots $\alpha \in \mathfrak{h}_{\mathbb{C}}^{*}$ are given by

$$
\alpha_{i j}(H)=h_{i}-h_{j}, \quad 1 \leqslant i, j \leqslant n, i \neq j,
$$

with $\alpha_{i j}$ positive if $i<j$. Furthermore,

$$
\begin{aligned}
& E_{\alpha_{i j}}=E_{i j}, \text { the elementary matrix with the }(i, j) \text { th entry equal to } 1, \\
& H_{\alpha_{i j}}=\operatorname{diag}\left(h_{1}, \ldots, h_{n}\right) \text { with } h_{i}=1, h_{j}=-1 \text { and } h_{k}=0 \text { for all } k \neq i, j .
\end{aligned}
$$

The subset

$$
\Lambda=\left\{\alpha_{1,1+p}, \ldots, \alpha_{p, 2 p}\right\},
$$

is a maximal set of strongly orthogonal roots (see below). For $\gamma_{j}=\alpha_{j, j+p}$ we get, in particular,

$$
\begin{aligned}
\sum_{\gamma_{j} \in \Lambda} E_{\gamma_{j}} & =\left(\begin{array}{ccc}
0 & \operatorname{Id}_{p} & 0 \\
0 & 0 & 0 \\
0 & 0 & 0
\end{array}\right) \subset \mathfrak{p}_{+}, \\
\sum_{\gamma_{j} \in \Lambda} E_{-\gamma_{j}} & =\left(\begin{array}{ccc}
0 & 0 & 0 \\
\operatorname{Id}_{p} & 0 & 0 \\
0 & 0 & 0
\end{array}\right) \subset \mathfrak{p}_{-}, \\
\sum_{\gamma_{j} \in \Lambda} X_{\gamma_{j}} & =\left(\begin{array}{ccc}
0 & \operatorname{Id}_{p} & 0 \\
\operatorname{Id}_{p} & 0 & 0 \\
0 & 0 & 0
\end{array}\right) \subset \mathfrak{a} \subset \mathfrak{p},
\end{aligned}
$$

where $\mathfrak{a}$ is a maximal Abelian subalgebra contained in $\mathfrak{p}$.

Two roots $\alpha, \beta \in \Psi$ are called strongly orthogonal if neither $\alpha-\beta$ nor $\alpha+\beta$ is a root and, by a theorem of Harish-Chandra (see [25, pp. 582-583]) there exists a (maximal) set $\Lambda=\left\{\gamma_{1}, \ldots, \gamma_{r}\right\} \subset \Psi^{\mathfrak{p}_{+}}$of $r=r_{\mathcal{X}}$ strongly orthogonal positive noncompact roots. The associated vectors $X_{\gamma_{j}} \in \mathfrak{p}, \gamma_{j} \in \Lambda$, span then a maximal Abelian subspace $\mathfrak{a}$ of $\mathfrak{p}$ over $\mathbb{R}$. The subspace $\exp (\mathfrak{a}) x_{\epsilon} \subset b_{\epsilon}(\mathcal{X}) \subset M_{\epsilon}$ is the image of

$$
\mathfrak{a}_{\epsilon}:=\left\{\sum_{j=1}^{r} \tanh \left(t_{j}\right) E_{\epsilon \gamma_{j}} \mid \gamma_{j} \in \Lambda, t_{j} \in \mathbb{R}\right\} \subset \mathfrak{p}_{\epsilon}
$$

under the map $\xi_{\epsilon}: \mathfrak{p}_{\epsilon} \rightarrow M_{\epsilon}$, so that

$$
\xi_{\epsilon}^{-1} \circ b_{\epsilon}\left(\exp (\mathfrak{a}) x_{0}\right)=\xi_{\epsilon}^{-1}\left(\exp (\mathfrak{a}) x_{\epsilon}\right)=\mathfrak{a}_{\epsilon}
$$

is clearly bounded. From the $K$-equivariance of $\xi_{\epsilon}^{-1}$ and the Cartan decomposition $G=K \exp (\mathfrak{a}) K$, we obtain the following description of the Harish-Chandra realization of $\mathcal{X}$ as a bounded symmetric domain

$$
\mathcal{D}_{\epsilon}=\xi_{\epsilon}^{-1} \circ b_{\epsilon}(\mathcal{X})=\left\{\operatorname{Ad}(k) \sum_{j=1}^{r} \tanh \left(t_{j}\right) E_{\epsilon \gamma_{j}} \mid k \in K, t_{j} \in \mathbb{R}\right\} \subset \mathfrak{p}_{\epsilon} .
$$

The Harish-Chandra realization induces, moreover, an isomorphism $\operatorname{Isom}(\mathcal{X})^{\circ} \cong \operatorname{Aut}\left(\mathcal{D}_{\epsilon}\right)^{\circ}$, which links the Riemannian structure of $\mathcal{X}$ with the complex structure of $\mathcal{D}_{\epsilon}$. 


\subsection{The Shilov boundary}

As bounded domains in $\mathbb{C}^{N}$, the Harish-Chandra realizations of $\mathcal{X}$ are equipped with certain structures.

Definition 2.6. The Shilov boundary $\check{S}$ of a bounded domain $\mathcal{D}$ in a complex vector space is the unique minimal closed subset of the topological boundary $\partial \mathcal{D}$ of $\mathcal{D}$ with the property that all functions $f$, continuous on $\overline{\mathcal{D}}$ and holomorphic on $\mathcal{D}$, satisfy $|f(x)| \leqslant$ $\max _{y \in \check{S}}|f(y)|$ for all $x \in \mathcal{D}$.

The Shilov boundary $\check{S}_{\epsilon}$ of the Harish-Chandra realization $\mathcal{D}_{\epsilon}$ is the $\operatorname{Aut}\left(\mathcal{D}_{\epsilon}\right)^{\circ}$-orbit of the point

$$
\epsilon i o_{\epsilon}:=\epsilon i \sum_{j=1}^{r} E_{\epsilon \gamma_{j}} \in \overline{\mathcal{D}_{\epsilon}} \subset \mathfrak{p}_{\epsilon}
$$

where $E_{\epsilon \gamma_{j}}$ are, as above, the root vectors associated to the strongly orthogonal roots $\gamma_{j} \in \Lambda$.

Example 2.7. The Shilov boundary of $\mathcal{D}_{p, q}$ is

$$
\check{S}_{p, q}=\left\{Z \in \operatorname{Mat}(q, p, \mathbb{C}) \mid \operatorname{Id}_{p}-Z^{*} Z=0\right\} \subset \mathfrak{p}_{-},
$$

which corresponds to the space of maximal isotropic subspaces

$$
\mathrm{Is}_{p, q}=\left\{L \in \operatorname{Gr}_{p}\left(\mathbb{C}^{n}\right)|\langle\cdot, \cdot\rangle|_{L}=0\right\} \subset \overline{\mathcal{X}}_{p, q}
$$

under the Borel embedding $\mathcal{X}_{p, q} \subset \mathrm{Gr}_{p}\left(\mathbb{C}^{n}\right)$.

2.3.1. Shilov boundary in the Borel embeddings. The description of the Shilov boundary $\xi_{\epsilon}\left(\check{S}_{\epsilon}\right)$ in the Borel embeddings $b_{\epsilon}(\mathcal{X})$ requires the introduction of the Cayley element, which is defined by

$$
c:=\exp \left(\frac{\pi}{4} i \sum_{j=1}^{r} X_{\gamma_{j}}\right) \in \mathbf{G}
$$

where the $X_{\gamma_{j}}$ 's are, as above, the real vectors in $\mathfrak{p}$ corresponding to the strongly orthogonal roots $\gamma_{j} \in \Lambda$.

Proposition 2.8. With the above notations we have:

(1) $\xi_{\epsilon}\left(\check{S}_{\epsilon}\right)=G c^{\epsilon 1}\left(x_{\epsilon}\right)$, where $c^{\epsilon 1} \in\left\{c, c^{-1}\right\}$;

(2) the parabolic subgroup

$$
\mathbf{Q}:=c K_{\mathbb{C}} P_{-} c^{-1} \cap c^{-1} K_{\mathbb{C}} P_{+} c
$$

is defined over $\mathbb{R}$ and

$$
\operatorname{Stab}_{G}\left(c^{\epsilon 1} x_{\epsilon}\right)=\mathbf{Q}(\mathbb{R}) .
$$


Proof. For the first statement of the proposition, see [29]; as for the second, let $\sigma$ be the conjugation of $\mathbf{G}$ with respect to the real structure $G$. Then $\sigma(c)=c^{-1}$ and $\sigma\left(K_{\mathbb{C}} P_{+}\right)=K_{\mathbb{C}} P_{-}$, which shows that $\mathbf{Q}$ is defined over $\mathbb{R}$. The last statement follows from the fact that

$$
\operatorname{Stab}_{G}\left(c^{\epsilon 1} x_{\epsilon}\right)=c^{\epsilon 1} K_{\mathbb{C}} P_{-\epsilon} c^{-\epsilon 1} \cap G=c^{-\epsilon 1} K_{\mathbb{C}} P_{\epsilon} c^{\epsilon 1} \cap G=\mathbf{Q} \cap G=\mathbf{Q}(\mathbb{R}),
$$

where the second equality is obtained by applying $\sigma$.

For our purposes, the drawback of this description is that, while the Shilov boundary $\check{S}_{\epsilon}$ is isomorphic as a $G$-space to the real points of the complex projective $\mathbb{R}$-variety $\mathbf{G} / \mathbf{Q}$, objects such as $M_{\epsilon}$ - in which $\xi_{\epsilon}\left(\check{S}_{\epsilon}\right)$ sits - and the G-action on $M_{\epsilon}$ are not defined over $\mathbb{R}$. We now remedy this by giving a suitable realization of $\mathbf{G} / \mathbf{Q}$.

2.3.2. Complexification of the Shilov boundary. Let $\mathbf{L}=\mathbf{G} \times \mathbf{G}$ be the direct product of the complex algebraic group $\mathbf{G}$ with itself. Let $\Sigma(g, h):=(\sigma(h), \sigma(g))$. Then the conjugation $\Sigma$ defines an $\mathbb{R}$-structure on $\mathbf{L}$ for which the parabolic subgroup $\mathbf{N}_{\epsilon}:=$ $K_{\mathbb{C}} P_{\epsilon} \times K_{\mathbb{C}} P_{-\epsilon}$ is defined over $\mathbb{R}$. Via the identification $\mathbf{L} / \mathbf{N}_{\epsilon} \cong M_{\epsilon} \times M_{-\epsilon}$ of complex varieties we endow $M_{\epsilon} \times M_{-\epsilon}$ with the corresponding $\mathbb{R}$-structure. The morphism

$$
\Delta: \mathbf{G} \longrightarrow \mathbf{L}, \quad g \mapsto(g, g),
$$

is defined over $\mathbb{R}$ and, in particular, $\mathbf{G}$ acts $\mathbb{R}$-algebraically on $M_{\epsilon} \times M_{-\epsilon}$.

For $p_{\epsilon}:=\left(c^{\epsilon}\left(x_{\epsilon}\right), c^{-\epsilon}\left(x_{-\epsilon}\right)\right)$ we have $p_{\epsilon} \in\left(M_{\epsilon} \times M_{-\epsilon}\right)(\mathbb{R}), \mathbf{Q}=\operatorname{Stab}_{\mathbf{Q}}\left(p_{\epsilon}\right)$, so that the orbit map provides an identification of $\mathbb{R}$-varieties

$$
\mathbf{G} / \mathbf{Q} \rightarrow \mathbf{G} p_{\epsilon} \subset M_{\epsilon} \times M_{-\epsilon} .
$$

Similarly, we endow the affine space $\mathfrak{p}_{\epsilon} \times \mathfrak{p}_{-\epsilon}$ with the $\mathbb{R}$-structure given by $\Sigma$ and obtain in this way an $\mathbb{R}$-isomorphism

$$
\mathfrak{p}_{\epsilon} \times \mathfrak{p}_{-\epsilon} \stackrel{\xi_{\epsilon} \times \xi_{-\epsilon}}{\longrightarrow} U_{\epsilon} \times U_{-\epsilon}
$$

of $\mathbb{R}$-varieties sending $G\left(i \epsilon O_{\epsilon},-i \epsilon O_{-\epsilon}\right)$ into $U_{\epsilon} \times U_{-\epsilon}$ with image $\mathbf{G} p_{\epsilon}(\mathbb{R})$. Let

$$
\imath_{\epsilon}: \check{S}_{\epsilon} \longrightarrow \mathbf{G} p_{\epsilon}(\mathbb{R}), \quad g i \epsilon O_{\epsilon} \mapsto g p_{\epsilon}
$$

denote the resulting identification.

\subsection{Hermitian triple product and Bergmann kernels}

We will now use the model of $\mathbf{G} / \mathbf{Q}$ as an $\mathbb{R}$-subvariety of $M_{\epsilon} \times M_{-\epsilon}$ described above in order to define an invariant rational function on $(\mathbf{G} / \mathbf{Q})^{3}$, called the complex Hermitian triple product, and relate it to the Bergmann kernel of the bounded domain via the embedding $\imath_{\epsilon}$. 
Remark 2.9. The reader interested in seeing the construction below realized in a family of examples, namely $\mathrm{SU}(p, q)$, is referred to [6, Sections 3 and 4].

To this end, let us start by considering the Zariski open subset of $\mathbf{G} \times \mathfrak{p}_{\epsilon}$ defined by

$$
V_{\epsilon}:=\left\{(g, z) \in \mathbf{G} \times \mathfrak{p}_{\epsilon} \mid g(\exp (z)) \in \mathcal{O}_{\epsilon}\right\}
$$

and the automorphy factor

$$
\mathcal{J}_{\epsilon}: V_{\epsilon} \longrightarrow K_{\mathbb{C}}, \quad(g, z) \mapsto \pi_{0}^{\epsilon}(g \exp (z))
$$

where $\pi_{0}^{\epsilon}$ is the projection on $\mathcal{O}_{\epsilon}$ defined in (2.1). Using now the projections $\pi_{\epsilon}^{\epsilon}$ also defined in (2.1) let us consider the map

$$
V_{\epsilon} \longrightarrow \mathfrak{p}_{\epsilon}, \quad(g, z) \mapsto g_{*} z:=\log \left(\pi_{\epsilon}^{\epsilon}(g \exp (z))\right)
$$

which partially defines a G-action by birational isomorphisms of the affine space $\mathfrak{p}_{\epsilon}$. Notice that, via $\xi_{\epsilon}, \mathfrak{p}_{\epsilon}$ is identified with a Zariski open subset $U_{\epsilon} \subseteq M_{\epsilon}$ (see Lemma 2.3) and, moreover, the partially defined $\mathbf{G}$-action on $\mathfrak{p}_{\epsilon}$ corresponds, again via $\xi_{\epsilon}$, to the action of $\mathbf{G}$ on $M_{\epsilon}$ "restricted" to $U_{\epsilon}$ (the fact that $U_{\epsilon}$ is not $\mathbf{G}$-invariant corresponds to the fact that the $\mathbf{G}$-action on $\mathfrak{p}_{\epsilon}$ is only partially defined).

Let us now consider the Zariski open subset of $\mathfrak{p}_{\epsilon} \times \mathfrak{p}_{-\epsilon}$ defined by

$$
\begin{aligned}
W_{\epsilon}: & =\left\{(z, w) \in \mathfrak{p}_{\epsilon} \times \mathfrak{p}_{-\epsilon} \mid \exp (-w)(\exp (z)) \in \mathcal{O}_{\epsilon}\right\} \\
& =\left\{(z, w) \in \mathfrak{p}_{\epsilon} \times \mathfrak{p}_{-\epsilon} \mid(\exp (-w), \exp (z)) \in V_{\epsilon}\right\}
\end{aligned}
$$

and the complex automorphy kernel

$$
\mathcal{K}_{\epsilon}: W_{\epsilon} \longrightarrow K_{\mathbb{C}}, \quad(z, w) \mapsto \mathcal{J}_{\epsilon}(\exp (-w), z)
$$

With a series of formal verifications we obtain the following

Lemma 2.10. Let $(z, w) \in W_{\epsilon}$ and $g \in G$ be such that $\left(g_{*} z, g_{*} w\right) \in W_{\epsilon},(g, z) \in V_{\epsilon}$ and $(g, w) \in V_{-\epsilon}$. Then:

(1) $\mathcal{K}_{\epsilon}\left(g_{*} z, g_{*} w\right)=\mathcal{J}_{-\epsilon}(g, w) \mathcal{K}_{\epsilon}(z, w) \mathcal{J}_{\epsilon}(g, z)^{-1}$; and

(2) $\mathcal{K}_{\epsilon}(\sigma(w), \sigma(z))=\sigma\left(\mathcal{K}_{\epsilon}(z, w)\right)^{-1}$.

Define now

$$
\begin{aligned}
k_{\epsilon}(z, w) & :=\operatorname{det}\left(\left.\operatorname{Ad} \mathcal{K}_{\epsilon}(z, w)\right|_{\mathfrak{p}_{\epsilon}}\right) \\
j_{\epsilon}(g, z) & :=\operatorname{det}\left(\left.\operatorname{Ad} \mathcal{J}_{\epsilon}(g, z)\right|_{\mathfrak{p}_{\epsilon}}\right) .
\end{aligned}
$$

Remark 2.11. For $(z, w) \in W_{\epsilon}$ and $(g, z) \in V_{\epsilon}$, we have that $k_{\epsilon}(z, w) \neq 0$.

The relevance for complex analysis of the above algebraic objects is the following 
Proposition 2.12. ([34]) Let $\mathcal{D}_{\epsilon} \subset \mathfrak{p}_{\epsilon}$ be the bounded domain realization of $\mathcal{X}$. Then its Bergmann kernel $k_{\mathcal{D}_{\epsilon}}: \mathcal{D}_{\epsilon} \times \mathcal{D}_{\epsilon} \rightarrow \mathbb{C}$ is given by

$$
k_{\mathcal{D}_{\epsilon}}\left(z_{1}, z_{2}\right)=C_{\epsilon} k_{\epsilon}\left(z_{1}, \sigma\left(z_{2}\right)\right)
$$

for some nonzero constant $C_{\epsilon}$. In particular, the Bergmann kernel $k_{\mathcal{D}_{\epsilon}}$ extends continuously to

$$
\overline{\mathcal{D}}_{\epsilon}^{(2)}:=\left\{\left(z_{1}, z_{2}\right) \in \overline{\mathcal{D}}_{\epsilon}^{2} \mid\left(z_{1}, \sigma\left(z_{2}\right)\right) \in W_{\epsilon}\right\}
$$

and is nowhere nonzero.

Lemma 2.10 now readily implies:

Lemma 2.13. Under the same hypotheses as Lemma 2.10 we have:

(1) $k_{\epsilon}\left(g_{*} z, g_{*} w\right)=j_{-\epsilon}(g, w)^{-1} k_{\epsilon}(z, w) j_{\epsilon}(g, z)^{-1}$; and

(2) $k_{\epsilon}(\sigma(w), \sigma(z))=\overline{k_{\epsilon}(z, w)}$

Example 2.14. Recall that for $\mathfrak{g}=\mathfrak{s u}(p, q)$ we have

$$
\mathfrak{p}_{+}=\operatorname{Mat}(p, q, \mathbb{C}), \quad \mathfrak{p}_{-}=\operatorname{Mat}(q, p, \mathbb{C}),
$$

with $\sigma(Z)=-Z^{*}$. Thus the real structure on $\mathfrak{p}_{-} \times \mathfrak{p}_{+}$is given by

$$
\Sigma: \mathfrak{p}_{-} \times \mathfrak{p}_{+} \longrightarrow \mathfrak{p}_{-} \times \mathfrak{p}_{+}, \quad(Z, W) \mapsto\left(-W^{*},-Z^{*}\right) .
$$

The automorphy kernel

$$
\mathcal{K}_{-}: \operatorname{Mat}(q, p, \mathbb{C}) \times \operatorname{Mat}(p, q, \mathbb{C}) \longrightarrow K_{\mathbb{C}}, \quad(Z, W) \mapsto\left(\begin{array}{ccc}
\operatorname{Id}_{p}-W Z & 0 & 0 \\
0 & \left(\operatorname{Id}_{p}-W Z\right)^{-1} & 0 \\
0 & 0 & 0
\end{array}\right),
$$

is well defined on the subset

$$
W_{-}=\left\{(Z, W) \in \operatorname{Mat}(q, p, \mathbb{C}) \times \operatorname{Mat}(p, q, \mathbb{C}) \mid \operatorname{det}\left(\operatorname{Id}_{p}-W Z\right) \neq 0\right\} .
$$

The Bergman kernel is

$$
k_{-}: \operatorname{Mat}(q, p, \mathbb{C}) \times \operatorname{Mat}(q, p, \mathbb{C}) \longrightarrow \mathbb{C}, \quad\left(Z_{1}, Z_{2}\right) \mapsto \operatorname{det}\left(\operatorname{Id}_{p}-Z_{2}^{*} Z_{1}\right)^{-n},
$$

where $n=2(p-1)+(q-p)+2=p+q$.

Let $A=\mathbb{C} \times \mathbb{C}$ be the algebra product of two copies of $\mathbb{C}$ endowed with the real structure defined by $\Sigma(a, b)=(\bar{b}, \bar{a})$. Then the algebra homomorphism

$$
\Delta: \mathbb{C} \longrightarrow A, \quad \lambda \mapsto(\lambda, \bar{\lambda}),
$$

is defined over $\mathbb{R}$ and $\Delta(\mathbb{C})=A(\mathbb{R})$. The algebra $A$ has $\mathbb{1}=(1,1)$ as unit and the subgroup $\mathbb{C}^{\times} \mathbb{1}=\{(\lambda, \lambda): \lambda \in \mathbb{C}\}$ is defined over $\mathbb{R}$.

$$
\begin{aligned}
\text { If } z_{j}=\left(z_{j}^{\epsilon}, z_{j}^{-\epsilon}\right) & \in \mathfrak{p}_{\epsilon} \times \mathfrak{p}_{-\epsilon} \text { for } j=1,2 \text { on } \\
W_{\epsilon}^{(2)} & :=\left\{\left(z_{1}, z_{2}\right) \in\left(\mathfrak{p}_{\epsilon} \times \mathfrak{p}_{-\epsilon}\right)^{2} \mid\left(z_{i}^{\epsilon}, z_{j}^{-\epsilon}\right) \in W_{\epsilon}, i \neq j\right\}
\end{aligned}
$$

we define

$$
F\left(z_{1}, z_{2}\right):=\left(k_{\epsilon}\left(z_{1}^{\epsilon}, z_{2}^{-\epsilon}\right), k_{\epsilon}\left(z_{2}^{\epsilon}, z_{1}^{-\epsilon}\right)\right) \in A^{\times}
$$

and on

$$
W_{\epsilon}^{(3)}=\left\{\left(z_{1}, z_{2}, z_{3}\right) \in\left(\mathfrak{p}_{\epsilon} \times \mathfrak{p}_{-\epsilon}\right)^{3} \mid\left(z_{i}, z_{j}\right) \in W_{\epsilon}^{(2)}, i \neq j\right\}
$$

we define

$$
\left\langle z_{1}, z_{2}, z_{3}\right\rangle_{\mathbb{C}}:=F\left(z_{1}, z_{2}\right) F\left(z_{2}, z_{3}\right) F\left(z_{3}, z_{1}\right) \in A^{\times} .
$$

Then using Lemma 2.13 one can verify the following 


\section{Lemma 2.15.}

(1) If $g \in \mathbf{G}$ and $\left(z_{1}, z_{2}, z_{3}\right) \in W_{\epsilon}^{(3)}$ are such that the triple $\left(g_{*} z_{1}, g_{*} z_{2}, g_{*} z_{3}\right)$ also belongs to $W_{\epsilon}^{(3)}$, then

$$
\left\langle g_{*} z_{1}, g_{*} z_{2}, g_{*} z_{3}\right\rangle_{\mathbb{C}} \in \mathbb{C}^{\times}\left\langle z_{1}, z_{2}, z_{3}\right\rangle_{\mathbb{C}} .
$$

(2) For any quadruple $\left(z_{1}, z_{2}, z_{3}, z_{4}\right)$ such that for $1 \leqslant i<j<k \leqslant 4$ we have $\left(z_{i}, z_{j}, z_{k}\right) \in W_{\epsilon}^{(3)}$, then

$$
\left\langle z_{2}, z_{3}, z_{4}\right\rangle_{\mathbb{C}}\left\langle z_{1}, z_{3}, z_{4}\right\rangle_{\mathbb{C}}^{-1}\left\langle z_{1}, z_{2}, z_{4}\right\rangle_{\mathbb{C}}\left\langle z_{1}, z_{2}, z_{3}\right\rangle_{\mathbb{C}}^{-1} \in \mathbb{C}^{\times} \mathbb{1} .
$$

(3) For $\left(z_{1}, z_{2}, z_{3}\right) \in W_{\epsilon}^{(3)}$ such that also $\left(\Sigma\left(z_{1}\right), \Sigma\left(z_{2}\right), \Sigma\left(z_{3}\right)\right) \in W_{\epsilon}^{(3)}$, then

$$
\left\langle\Sigma\left(z_{1}\right), \Sigma\left(z_{2}\right), \Sigma\left(z_{3}\right)\right\rangle_{\mathbb{C}}=\Sigma\left(\left\langle z_{1}, z_{2}, z_{3}\right\rangle_{\mathbb{C}}\right) \text {. }
$$

We summarize the essential points of what we obtained so far in the following

Corollary 2.16. The algebraic subgroup $\mathbb{C}^{\times} \mathbb{1}$ of $A^{\times}$is defined over $\mathbb{R}$ and the map

$$
\langle\langle\cdot, \cdot, \cdot\rangle\rangle_{\mathbb{C}}: W_{\epsilon}^{(3)} \longrightarrow \mathbb{C}^{\times} \mathbb{1} \backslash A^{\times},
$$

obtained from (2.4) by passing to the quotient of $A^{\times}$by $\mathbb{C}^{\times} \mathbf{1}$, is a $\mathbf{G}$-invariant regular map defined over $\mathbb{R}$.

Using now the $\mathbb{R}$-isomorphism

$$
\xi_{\epsilon} \times \xi_{-\epsilon}: \mathfrak{p}_{\epsilon} \times \mathfrak{p}_{-\epsilon} \longrightarrow U_{\epsilon} \times U_{-\epsilon}
$$

we denote again by $\langle\langle\cdot, \cdot, \cdot\rangle\rangle_{\mathbb{C}}$ the rational map on $\left(M_{\epsilon} \times M_{-\epsilon}\right)^{3}$ with values in $\mathbb{C}^{\times} \mathbb{1} \backslash A^{\times}$which (Lemma 2.15(1)) can be defined on the union of the G-translates of $\left(\xi_{\epsilon} \times \xi_{-\epsilon}\right)^{3}\left(W_{\epsilon}^{(3)}\right)$. Considering now $\mathbf{G} / \mathbf{Q}$ as an $\mathbb{R}$-subvariety of $M_{\epsilon} \times M_{-\epsilon}$ as in Section 2.4 , we restrict $\langle\langle\cdot, \cdot, \cdot\rangle\rangle_{\mathbb{C}}$ to a rational map on $(\mathbf{G} / \mathbf{Q})^{3}$ - which we call the complex Hermitian triple product-defined over $\mathbb{R}$ (Lemma 2.13(3)) and G-invariant (Lemma 2.13(2)).

We now establish the connection with the Bergmann kernel of $\mathcal{D}_{\epsilon}$. According to Proposition 2.12, the map

$$
\langle\cdot, \cdot,\rangle: \overline{\mathcal{D}}_{\epsilon}^{(3)} \longrightarrow \mathbb{C}^{\times}
$$

given by

$$
\left\langle z_{1}, z_{2}, z_{3}\right\rangle:=k_{\mathcal{D}_{\epsilon}}\left(z_{1}, z_{2}\right) k_{\mathcal{D}_{\epsilon}}\left(z_{2}, z_{3}\right) k_{\mathcal{D}_{\epsilon}}\left(z_{3}, z_{1}\right)
$$

where

$$
\overline{\mathcal{D}}_{\epsilon}^{(3)}=\left\{\left(z_{1}, z_{2}, z_{3}\right) \in \mathfrak{p}_{\epsilon}^{3} \mid\left(z_{i}, z_{j}\right) \in \overline{\mathcal{D}}_{\epsilon}^{(2)}, i \neq j\right\},
$$

is continuous and nowhere vanishing, and its composition $\langle\langle\cdot, \cdot, \cdot\rangle\rangle$ with the projection $\mathbb{C}^{\times} \rightarrow \mathbb{R}^{\times} \backslash \mathbb{C}^{\times}$is $G$-invariant (Lemma $2.15(2)$ ). We call $\langle\langle\cdot, \cdot, \cdot\rangle\rangle$ the Hermitian triple product. Recall now that as a homogeneous space, the Shilov boundary $\check{S}_{\epsilon}$ is isomorphic to $G / Q$, where $Q=\mathbf{Q}(\mathbb{R})$ is thus a real parabolic subgroup of $G$. Thus let $\check{S}_{\epsilon}^{(2)}$ denote the unique open $G$-orbit in $\check{S}_{\epsilon}^{2}$ and

$$
\check{S}_{\epsilon}^{(3)}:=\left\{\left(z_{1}, z_{2}, z_{3}\right) \in \check{S}_{\epsilon}^{3} \mid\left(z_{i}, z_{j}\right) \in \check{S}_{\epsilon}^{(2)}, i \neq j\right\} .
$$

Then $\overline{\mathcal{D}}_{\epsilon}^{(3)} \supset \check{S}_{\epsilon}^{(3)}$ and the complex Hermitian triple product is defined on $\imath_{\epsilon}^{3}\left(\check{S}_{\epsilon}^{(3)}\right) \subset$ $(\mathbf{G} / \mathbf{Q})^{3}$, where $\imath_{\epsilon}: \check{S}_{\epsilon} \rightarrow \mathbf{G} / \mathbf{Q}$ is the embedding defined in Section 2.3.2 identifying $\check{S}_{\epsilon}$ with $(\mathbf{G} / \mathbf{Q})(\mathbb{R})$. Denoting again by $\Delta: \mathbb{R}^{\times} \backslash \mathbb{C}^{\times} \rightarrow \mathbb{C}^{\times} \mathbb{1} \backslash A^{\times}$the homomorphism induced by $\Delta: \mathbb{C}^{\times} \rightarrow A^{\times}$we obtain, from Proposition 2.12 : 
Corollary 2.17. The diagram

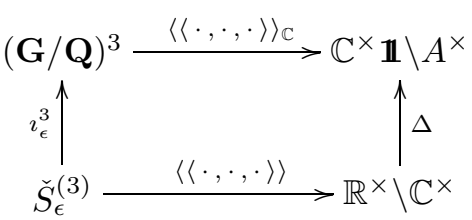

commutes.

\section{Characterization of domains not of tube type}

Keeping the notations of Section 2 we will assume throughout this section that $\mathcal{X}$ is an irreducible Hermitian symmetric space and denote, for ease of notation, $\mathcal{D}=\mathcal{D}_{+} \subset \mathfrak{p}_{+}$ its Harish-Chandra realization in $\mathfrak{p}_{+}$as well as $\check{S}=\check{S}_{+}$its Shilov boundary. Our main objective is to prove Theorem 1 in the Introduction.

\subsection{Bruhat cell decomposition}

The equivalence of the properties (1) and (2) in Theorem 1 will follow from the determination of the dimensions of the Bruhat cells in $G / Q$ which is the subject matter of this subsection. To this end we need the precise form of the restricted root system of $\mathfrak{g}$ and the description of $Q$ in these terms. Recall that $\Lambda=\left\{\gamma_{1}, \ldots, \gamma_{r}\right\}$ is the set of strongly orthogonal roots in Section 2.2; let $\mathfrak{h}^{-}$be the real span of the vectors $i H_{\gamma_{i}}$ for $\gamma_{i} \in \Lambda$. Then $i \mathfrak{h}^{-}$is a Cartan subalgebra of $\mathfrak{g}^{c}:=\operatorname{Ad}(c) \mathfrak{g}$, where $c$ is the Cayley element defined in Section 2.3.1 and the restricted root system $\Xi=\Xi\left(\mathfrak{g}^{c}, i \mathfrak{h}^{-}\right)$of $\mathbb{R}$-roots of $i \mathfrak{h}^{-}$ in $\mathfrak{g}^{c}$ is one of the following two types:

$\star$ Type $\mathrm{C}_{r}$ (tube type), namely,

$$
\Xi=\left\{ \pm \frac{1}{2} \gamma_{i} \pm \frac{1}{2} \gamma_{j}, i \neq j ; \pm \gamma_{i}\right\},
$$

where the roots $\pm \gamma_{i}$ have multiplicity 1 ; for later purposes, we let $a \geqslant 1$ denote the common multiplicity of the roots $\pm \frac{1}{2} \gamma_{i} \pm \frac{1}{2} \gamma_{j}$;

$\star$ Type $\mathrm{BC}_{r}$ (not of tube type), namely,

$$
\Xi=\left\{ \pm \frac{1}{2} \gamma_{i} \pm \frac{1}{2} \gamma_{j}, i \neq j ; \pm \gamma_{i} ; \pm \frac{1}{2} \gamma_{j}\right\},
$$

where $\pm \frac{1}{2} \gamma_{j}$ are of even multiplicity, denoted by $2 b$.

Example 3.1. The multiplicities for $\mathfrak{s u}(p, q), 1 \leqslant p \leqslant q$, are $r=p, a=2, b=q-p$. In particular, $\mathcal{X}_{p q}$ is of tube type if and only if $p=q$.

A set of positive simple roots is given by

$$
\Delta_{i \mathfrak{h}^{-}}=\left\{\frac{1}{2} \gamma_{i}-\frac{1}{2} \gamma_{i+1}, 1 \leqslant i \leqslant r-1 ; \delta \gamma_{r}\right\}
$$

where $\delta=1$ in the type $\mathrm{C}_{r}$ case and $\delta=\frac{1}{2}$ in the type $\mathrm{BC}_{r}$ case.

For our purposes we apply $\operatorname{Ad}(c)$ to this situation; this automorphism interchanges $i \mathfrak{h}^{-}$and $\operatorname{ad}\left(Z_{0}\right) \mathfrak{a}$ in $\mathfrak{g}_{\mathbb{C}}$ and sends the root system $\Xi$ to the root system $\Xi^{\prime}$ given by the 
$\mathbb{R}$-roots of $\operatorname{ad}\left(Z_{0}\right) \mathfrak{a}$ in $\mathfrak{g}$. Denoting by $\mu_{j}$ the image of $\gamma_{j}$, the set of simple negative roots becomes

$$
\Delta=\left\{-\frac{1}{2} \mu_{i}+\frac{1}{2} \mu_{i+1}, 1 \leqslant i \leqslant r-1 ;-\delta \mu_{r}\right\} .
$$

Recall that the standard real parabolic subgroups $P_{\Theta}$ of $G$ correspond to subsets $\Theta$ of $\Delta$ with the convention that $P:=P_{\emptyset}$ is minimal parabolic. For the parabolic subgroup $Q=G \cap c K_{\mathbb{C}} P_{-} c^{-1}$ we have the well known

Lemma 3.2. ([29, Theorem 5.9], [28, § IV.2.8]) If $\Theta=\Delta \backslash\left\{-\delta \mu_{r}\right\}$, then $Q=P_{\Theta}$.

The Weyl group $W$ of the restricted root system is generated by the reflections $s_{1}, \ldots, s_{r}$, where, for $1 \leqslant i \leqslant r-1, s_{i}$ is the reflection in $-\frac{1}{2} \mu_{i}+\frac{1}{2} \mu_{i+1}$, and $s_{r}$ is the reflection in $-\delta \mu_{r}$. Let $W_{\Theta}$ be the subgroup generated by $\left\{s_{1}, \ldots, s_{r-1}\right\}$ and $W^{\Theta}$ a set of representatives (to be determined below) of $W$ modulo $W_{\Theta}$. The Bruhat decomposition is the following disjoint union

$$
G / Q=\bigcup_{w \in W^{\ominus}} c(w)
$$

where $c(w)=P w Q$.

Lemma 3.3. Let $t_{k}:=s_{k} \ldots s_{r}, 1 \leqslant k \leqslant r$. Then $W^{\Theta}:=\left\{t_{1}, t_{2} t_{1}, \ldots, t_{r} \ldots t_{1}\right\}$ is a set of representatives of $W$ modulo $W_{\Theta}$.

Proof. This follows from the explicit form of the action of $t_{k}$ on the root system. Namely,

$$
\begin{aligned}
& t_{k}\left(\mu_{i}\right)=\mu_{i}, \quad \text { for } 1 \leqslant i<k, \\
& t_{k}\left(\mu_{i}\right)=\mu_{i+1}, \quad \text { for } k \leqslant i \leqslant r-1, \\
& t_{k}\left(\mu_{r}\right)=-\mu_{k} . \quad \square
\end{aligned}
$$

Lemma 3.4. Let $w_{m}=t_{r} \ldots t_{1}$. Then:

(1) $c\left(w_{m}\right)=Q w_{m} Q$ is open and of dimension $(2 b+1) r+\operatorname{ar}(r-1) / 2$;

(2) the codimension of $c\left(t_{r-k} \ldots t_{1}\right)$ in $G / Q$ is equal to $(2 b+1) k+a k(k-1) / 2$.

Proof. (1) The dimension is simply the sum of the multiplicities of the positive roots.

(2) Let $\Psi$ be the set of all nondivisible negative roots in $\Xi^{\prime}$ which do not lie in the span of $\Theta$. For $w \in W^{\Theta}$ define

$$
\Xi_{w}:=w(\Psi) \cap \Xi^{+},
$$

where $\Xi^{+}$is the set of positive roots in $\Xi^{\prime}$; let

$$
\mathfrak{g}_{w}=\sum_{\alpha \in \Xi_{w}}\left(\mathfrak{g}_{\alpha}+\mathfrak{g}_{2 \alpha}\right)
$$

and let $U_{w}$ be the unipotent subgroup corresponding to $\mathfrak{g}_{w}$. Then

is a diffeomorphism and, consequently,

$$
U_{w} \longrightarrow c(w), \quad u \mapsto u w Q,
$$

$$
\operatorname{dim} c(w)=\operatorname{dim} U_{w}=\operatorname{dim} \mathfrak{g}_{w} .
$$

To prove (2) it suffices then to check that

$$
\Xi_{t_{r} \ldots t_{1}}-\Xi_{t_{r-k} \ldots t_{1}}=\left\{\eta \mu_{r}, \ldots, \eta \mu_{r-k+1} ; \frac{1}{2} \mu_{i}+\frac{1}{2} \mu_{j}, j>i \geqslant r-k+1\right\},
$$

where $\eta=1$ in the case $\mathrm{C}_{r}$ and $\eta \in\left\{1, \frac{1}{2}\right\}$ in the case $\mathrm{BC}_{r}$, which, together with the multiplicities, gives the formula for the codimension.

Lemma 3.4 then implies: 
Corollary 3.5. Let $\mathcal{X}$ be an irreducible Hermitian symmetric space:

(1) if $\mathcal{X}$ is of tube type, then

$$
\operatorname{codim} c\left(t_{r-1} \ldots t_{1}\right)=1
$$

(2) if $\mathcal{X}$ is not of tube type, then

$$
\operatorname{codim} c\left(t_{r-k} \ldots t_{1}\right) \geqslant 3 \text { for } 1 \leqslant k \leqslant r .
$$

Corollary 3.6. If $\mathcal{X}$ is not of tube type, then $\check{S}^{(3)}$ is connected.

Proof. Indeed, $\check{S}^{(3)}$ is obtained from $\check{S}^{3}$ by removing finitely many subsets of codimension at least 3 (Corollary 3.5(2)).

\subsection{The Bergmann cocycle}

The equivalence of the properties (1), (3) and (4) in Theorem 1 rests on the relation between the Hermitian triple product and the Kähler area of geodesic triangles in $\mathcal{D}$, and relies on the results of Clerc and Ørsted.

Let $k_{\mathcal{D}}: \mathcal{D} \times \mathcal{D} \rightarrow \mathbb{C}^{\times}$be the Bergmann kernel which, as previously noticed, extends continuously to $k_{\mathcal{D}}: \overline{\mathcal{D}}^{(2)} \rightarrow \mathbb{C}^{\times}$, and let $\arg k_{\mathcal{D}}: \overline{\mathcal{D}}^{(2)} \rightarrow \mathbb{R}$ denote the continuous determination of the argument of $k_{\mathcal{D}}$ vanishing on the diagonal of $\mathcal{D}^{2}$. Recall that the Kähler form of the Bergmann metric is given by

$$
\omega_{\text {Berg }}=i \partial \bar{\partial} \log k_{\mathcal{D}}(z, z) .
$$

The following is then central for our immediate and subsequent purposes:

Theorem 3.7. ([18], [17]) Let $\Delta(x, y, z) \subset \mathcal{D}$ be a smooth oriented triangle with geodesic sides and vertices $x, y, z \in \mathcal{D}$. Then

$$
\int_{\Delta(x, y, z)} \omega_{\operatorname{Berg}}=-\left[\arg k_{\mathcal{D}}(x, y)+\arg k_{\mathcal{D}}(y, z)+\arg k_{\mathcal{D}}(z, x)\right]
$$

for all $x, y, z \in \mathcal{D}$.

For $(x, y, z) \in \overline{\mathcal{D}}^{(3)}$ we define, as we may,

$$
\beta(x, y, z)=-\left[\arg k_{\mathcal{D}}(x, y)+\arg k_{\mathcal{D}}(y, z)+\arg k_{\mathcal{D}}(z, x)\right]
$$

and we conclude readily using Theorem 3.7 and the fact that $\mathcal{D}^{3}$ is dense in $\overline{\mathcal{D}}^{(3)}$ :

Corollary 3.8. The function

$$
\beta: \overline{\mathcal{D}}^{(3)} \longrightarrow \mathbb{R}
$$

is continuous, G-invariant, alternating and satisfies the cocycle identity

$$
d \beta\left(x_{1}, x_{2}, x_{3}, x_{4}\right)=0
$$

whenever $\left(x_{i}, x_{j}, x_{k}\right) \in \overline{\mathcal{D}}^{(3)}, i<j<k$.

We call $\beta$ the Bergmann cocycle, and remark that it satisfies the following relation with the Hermitian triple product

$$
\langle\langle x, y, z\rangle\rangle \equiv e^{i \beta(x, y, z)} \bmod \mathbb{R}^{\times}
$$

for $(x, y, z) \in \overline{\mathcal{D}}^{(3)}$.

Another important consequence of Theorem 3.7 is the following 
Corollary 3.9. ([17, Theorem 3.1]) For $(x, y, z) \in \mathcal{D}^{3}$, we have that

$$
|\beta(x, y, z)|<\pi r \frac{p_{G}}{2}
$$

and

$$
\sup _{(x, y, z) \in \check{S}(3)}|\beta(x, y, z)|=\pi r \frac{p_{G}}{2},
$$

where $p_{G}=(r-1) a+b+2$.

In fact, it is easy to see that the supremum is achieved when $x, y, z \in \check{S}$ are the vertices of a maximal triangle, that is, they are distinct and lie in the boundary of a diagonal holomorphic disk in a maximal polydisk. The fact that those are exactly the triples achieving the supremum is also due to Clerc and Ørsted, but we will not use it here.

Corollary 3.10. Let $\mathcal{X}$ be an irreducible Hermitian symmetric space.

(1) $([16$, Theorem 4.3]). If $\mathcal{X}$ is of tube type, then

$$
\beta\left(\check{S}^{(3)}\right)=\left\{-\pi r \frac{p_{G}}{2},-\pi(r-2) \frac{p_{G}}{2}, \ldots, \pi(r-2) \frac{p_{G}}{2}, \pi r \frac{p_{G}}{2}\right\} .
$$

(2) If $\mathcal{X}$ is not of tube type, then

$$
\beta\left(\check{S}^{(3)}\right)=\left[-\pi r \frac{p_{G}}{2}, \pi r \frac{p_{G}}{2}\right] .
$$

Proof. Part (1) follows from [16, Theorem 4.3].

(2) According to Corollary $3.9, \beta\left(\check{S}^{(3)}\right) \subset\left[-\pi r p_{G} / 2, \pi r p_{G} / 2\right]$; moreover, if $x, y, z \in \check{S}$ are the vertices of a maximal triangle which is positively oriented, then both $(x, y, z) \in$ $\check{S}^{(3)}$ and $(y, x, z) \in \check{S}^{(3)}$, with $\beta(x, y, z)=\pi r p_{G} / 2$ and $\beta(y, x, z)=-\pi r p_{G} / 2$. Moreover, since $\mathcal{X}$ is not of tube type, $\breve{S}^{(3)}$ is connected, which implies the assertion.

Example 3.11. For explicit computations which give the statement of Corollary 3.10 when $\mathcal{X}=\mathcal{X}_{p, q}$ we refer the reader to [6, Lemma 4.1].

Proof of Theorem 1. The implication $(1) \Rightarrow(2)$ is Corollary 3.6, the implication $(2) \Rightarrow$ (1) is Corollary $3.10(1)$, while the implication $(1) \Rightarrow(3)$ is Corollary $3.10(2)$. The equivalence of (3) and (4) follows from the fact that $\check{S}^{(3)}$ is Zariski dense in $(\mathbf{G} / \mathbf{Q})^{3}$.

To see that (3) implies (1) let us assume that $\mathcal{X}$ is of tube type. Then again from Corollary $3.10(2)$, if $(x, y, z) \in \check{S}^{(3)}$, we have that $\beta(x, y, z)=\pi(r-2 k) p_{G} / 2$ for $k \in \mathbb{N}$, where now $p_{G}=(r-1) a+2$. Since

$$
\frac{p_{G}}{2} r=\frac{r(r-1) a}{2}+r \in \mathbb{Z}
$$

and, consequently, $(r-2 k) p_{G} / 2 \in \mathbb{Z}$, we have that

$$
e^{-i \beta(x, y, z)} \in\{ \pm 1\} \quad \text { for all }(x, y, z) \in \check{S}^{(3)},
$$

and hence $\langle\langle x, y, z\rangle\rangle=e^{i \beta(x, y, z)} \equiv 1 \bmod \mathbb{R}^{\times}$. 


\section{The Kähler class, the bounded Kähler class and boundaries}

\subsection{The Kähler class is integral}

We keep the notation of Section 3, in particular, $\omega_{\text {Berg }} \in \Omega^{2}(\mathcal{D})^{G}$ is the BergmannKähler form of the Bergmann metric of $\mathcal{D}=\mathcal{D}_{+} \subset \mathfrak{p}_{+}$and define

$$
c_{\omega_{\text {Berg }}}\left(g_{1}, g_{2}, g_{3}\right):=\frac{1}{2 \pi} \int_{\Delta\left(g_{1} 0, g_{2} 0, g_{3} 0\right)} \omega_{\text {Berg }},
$$

where, as before, $\Delta(x, y, z) \subset \mathcal{D}$ denotes a smooth triangle with geodesic sides and vertices $x, y, z \in \mathcal{D}$. Then $c_{\omega_{\text {Berg }}}$ is a continuous $G$-invariant homogeneous cocycle on $G$ and defines a continuous class $\kappa_{G, \mathrm{~B}} \in \mathrm{H}_{\mathrm{c}}^{2}(G, \mathbb{R})$ called the Kähler class.

If $L$ is a locally compact group, let $\mathrm{H}_{\mathrm{c}}^{\bullet}(L, \mathbb{Z})$ denote its continuous cohomology with coefficients in $\mathbb{Z}$ which is computed, as is well known, using Borel cochains on $L$ with values in $\mathbb{Z}$ (see [33]). Let

$$
\bigotimes_{\mathbb{R}}: \mathrm{H}_{\mathrm{c}}^{\bullet}(L, \mathbb{Z}) \longrightarrow \mathrm{H}_{\mathrm{c}}^{\bullet}(L, \mathbb{R})
$$

denote the change of coefficients, and consider now the diagram

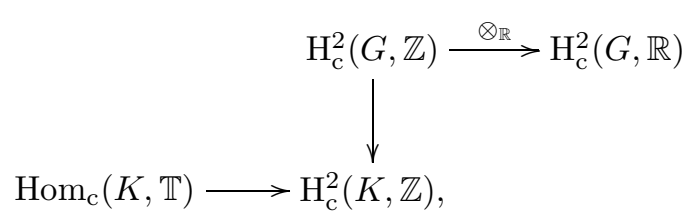

where the map corresponding to the vertical arrow is the restriction to $K$, and that to the bottom one is the connecting homomorphism coming from the short exact sequence of coefficients

$$
0 \longrightarrow \mathbb{Z} \longrightarrow \mathbb{R} \stackrel{E}{\longrightarrow} \mathbb{T} \longrightarrow 0
$$

where $\mathbb{T}$ is the circle group and $E(x):=\exp (2 \pi i x)$. It is well known that both of these maps are isomorphisms [24], so that we may speak of a class in $\mathrm{H}_{\mathrm{c}}^{2}(G, \mathbb{R})$ corresponding to a continuous homomorphism $K \rightarrow \mathbb{T}$ in $\operatorname{Hom}_{\mathrm{c}}(K, \mathbb{T})$. Now let $j: K \rightarrow \mathbb{T}$ be the continuous homomorphism which associates to $k \in K$ the complex Jacobian of $z \mapsto k z$ at the fixed point $0 \in \mathcal{D}$. Then we have:

Proposition 4.1. The Kähler class $\kappa_{G, \mathrm{~B}} \in \mathrm{H}_{\mathrm{c}}^{2}(G, \mathbb{R})$ corresponds to the complex Jacobian $j \in \operatorname{Hom}_{\mathrm{c}}(K, \mathbb{T})$. In particular, $\kappa_{G, \mathrm{~B}}$ is in the image of $\bigotimes_{\mathbb{R}}: \mathrm{H}_{\mathrm{c}}^{2}(G, \mathbb{Z}) \rightarrow \mathrm{H}_{\mathrm{c}}^{2}(G, \mathbb{R})$.

Proof. Let $u \in \operatorname{Hom}_{\mathrm{c}}(K, \mathbb{T})$ and let $\Omega_{u}$ be the $G$-invariant two-form on $\mathcal{D}$ whose value at $T_{0} \mathcal{D} \cong \mathfrak{p}$ is given by

$$
\left(\Omega_{u}\right)_{0}(X, Y)=\frac{1}{4 \pi i}\left(D_{e} u\right)([X, Y])
$$

where $D_{e} u: \mathfrak{k} \rightarrow \mathbb{R}$ is the derivative at $e$ of $u$. Then, according to [20], a continuous cocycle $c_{\Omega_{u}}: G^{3} \rightarrow \mathbb{R}$ representing the class in $\mathrm{H}_{\mathrm{c}}^{2}(G, \mathbb{R})$ corresponding to $u$ is given by

$$
c_{\Omega_{u}}\left(g_{1}, g_{2}, g_{3}\right):=\frac{1}{2 \pi} \int_{\Delta\left(g_{1} 0, g_{2} 0, g_{3} 0\right)} \Omega_{u} .
$$


Observing that the Bergmann metric is $\frac{1}{2}$ of the metric given by the Killing form, it follows from $[6,(5.4)]$ that

$$
\left(D_{e} u\right)([X, Y])=\frac{2}{\operatorname{dim} \mathfrak{p}}\left(D_{e} u\right)\left(Z_{0}\right)\left(\omega_{\mathrm{Berg}}\right)_{0}(X, Y),
$$

where $\left(\omega_{\text {Berg }}\right)_{0}$ is the value at 0 of the Kähler form $\omega_{\text {Berg }}$ and, consequently

$$
\left(\Omega_{u}\right)_{0}(X, Y)=\frac{1}{4 \pi i} \frac{2}{\operatorname{dim} \mathfrak{p}}\left(D_{e} u\right)\left(Z_{0}\right)\left(\omega_{\mathrm{Berg}}\right)_{0}(X, Y)
$$

We now apply this to $u=j$, where by definition we have that

$$
j\left(\exp \left(t Z_{0}\right)\right)=\operatorname{det}\left(\left.\operatorname{Ad}\left(\exp \left(t Z_{0}\right)\right)\right|_{\mathfrak{p}_{+}}\right)
$$

Taking into account that $\operatorname{Ad}\left(\exp \left(t Z_{0}\right)\right)$ acts on $\mathfrak{p}_{+}$as multiplication by $e^{i t}$, we get that

$$
j\left(\exp \left(t Z_{0}\right)\right)=e^{i t \operatorname{dim} \mathfrak{p}}
$$

and thus $\left(D_{e} u\right)\left(Z_{0}\right)=i \operatorname{dim} \mathfrak{p}$, which shows that $\left(\Omega_{u}\right)_{0}=\left(\omega_{\text {Berg }}\right)_{0}$, thus concluding the proof.

\subsection{The bounded Kähler class}

The cocycle $c_{\omega_{\text {Berg }}}$ is related to the Bergmann cocycle defined in (3.1) by the relation

$$
c_{\omega_{\mathrm{Berg}}}\left(g_{1}, g_{2}, g_{3}\right)=\frac{1}{2 \pi} \beta\left(g_{1} 0, g_{2} 0, g_{3} 0\right)
$$

so that Corollary 3.8 implies that

$$
\left\|c_{\omega_{\text {Berg }}}\right\|_{\infty} \leqslant \frac{p_{G} r}{4}
$$

Thus $c_{\omega_{\mathrm{Berg}}}$ gives a bounded continuous class $\kappa_{G, \mathrm{~B}}^{\mathrm{b}} \in \mathrm{H}_{\mathrm{cb}}^{2}(G, \mathbb{R})$, called the bounded Kähler class, which corresponds to the Kähler class $\kappa_{G, \mathrm{~B}}$ via the comparison map

$$
\mathrm{H}_{\mathrm{cb}}^{2}(G, \mathbb{R}) \longrightarrow \mathrm{H}_{\mathrm{c}}^{2}(G, \mathbb{R})
$$

which in this case is an isomorphism [10]. Let now $\mathcal{B}_{\text {alt }}^{\infty}\left(\check{S}^{n}\right)$ denote the Banach $G$-module of bounded Borel cochains on $\check{S}^{n}$ equipped with the simplicial coboundary operator

$$
d: \mathcal{B}_{\text {alt }}^{\infty}\left(\check{S}^{n}\right) \longrightarrow \mathcal{B}_{\text {alt }}^{\infty}\left(\check{S}^{n+1}\right) .
$$

Then [5]

$$
\mathbb{R} \longrightarrow \mathrm{L}^{\infty}(\check{S}) \longrightarrow \mathrm{L}_{\text {alt }}^{\infty}\left(\check{S}^{2}\right) \longrightarrow \ldots
$$

is a strong $G$-resolution of the trivial $G$-module $\mathbb{R}$ and we have a natural map

$$
\mathrm{H}^{\bullet}\left(\mathcal{B}_{\mathrm{alt}}^{\infty}\left(\check{S}^{\bullet}\right)^{G}\right) \longrightarrow \mathrm{H}_{\mathrm{cb}}^{\bullet}(G, \mathbb{R})
$$

We now use the following 
Theorem 4.2. ([14]) The cocycle $\beta: \check{S}^{(3)} \rightarrow \mathbb{R}$ extends to a $G$-invariant alternating Borel cocycle on $\tilde{S}^{3}$ such that

$$
\sup _{(x, y, z) \in \check{S}^{(3)}}|\beta(x, y, z)|=\pi r \frac{p_{G}}{2} .
$$

Let us denote by

$$
\beta_{\check{S}}: \check{S}^{3} \longrightarrow \mathbb{R}
$$

the extension of $\beta$ in Theorem 4.2 and by $\left[\beta_{\check{S}}\right] \in \mathrm{H}^{2}\left(\mathcal{B}_{\text {alt }}^{\infty}\left(\check{S}^{\bullet}\right)^{G}\right)$ its class. Then we have:

Proposition 4.3. The class $\left[\beta_{\breve{S}}\right]$ goes to $2 \pi \kappa_{G, \mathrm{~B}}^{\mathrm{b}}$ under the natural map in (4.1).

Proof. Denote for simplicity by $b:=i o_{+} \in \check{S}$ the point for which $\operatorname{Stab}_{G}(b)=Q$. Then we have morphisms of complexes

$$
\mathcal{B}_{\mathrm{alt}}^{\infty}\left(\check{S}^{\bullet}\right) \longrightarrow \mathrm{L}^{\infty}\left(\check{S}^{\bullet}\right) \longrightarrow \mathrm{L}_{\mathrm{alt}}^{\infty}\left(G^{\bullet}\right),
$$

where the second morphism is induced by the orbital map

$$
G \longrightarrow \check{S}, \quad g \mapsto g b .
$$

The resulting morphism $\mathcal{B}_{\text {alt }}^{\infty}\left(\check{S}^{\bullet}\right) \rightarrow \mathrm{L}_{\text {alt }}^{\infty}\left(G^{\bullet}\right)$ of complexes extends the identity $\mathbb{R} \rightarrow \mathbb{R}$ and, consequently, [11], [32], the map induced in cohomology implements the natural map (4.1). To finish the proof it then suffices to show that the bounded cocycle $\bar{c} \in$ $\mathrm{L}_{\text {alt }}^{\infty}\left(G^{3}\right)$ defined by

$$
\bar{c}\left(g_{1}, g_{2}, g_{3}\right):=\frac{1}{2 \pi} \beta_{\breve{S}}\left(g_{1} b, g_{2} b, g_{3} b\right)
$$

is cohomologous to $c_{\omega_{\text {Berg }}}$ in $\left(\mathrm{L}_{\text {alt }}^{\infty}\left(G^{\bullet}\right), d^{\bullet}\right)$. This is formal and rests on the cocycle property of $\beta$ on $\overline{\mathcal{D}}^{(3)}$. Namely, if $g, h \in G$ with $(g b, h b) \in \check{S}^{(2)}$, define

$$
\gamma(g, h):=\beta(g b, h b, h o)+\beta(h o, g o, g b) .
$$

Then $\gamma \in \mathrm{L}^{\infty}(G \times G)^{G}$ and one verifies that

$$
c_{\omega_{\mathrm{Berg}}}=\bar{c}+d \gamma
$$

almost everywhere.

\subsection{A formula for the pullback}

Let $H$ be a locally compact second countable group. Following Kaimanovich (slightly extending a result in [11]), there is an étalée probability measure on $H$ such that, if $(B, \nu)$ denotes the corresponding Poisson boundary, we have: 
Proposition 4.4. ([27]) The diagonal H-action on $(B \times B, \nu \times \nu)$ is ergodic.

At any rate, the $H$-action on $(B, \nu)$ is amenable and thus the complex

$$
\mathbb{R} \longrightarrow \ldots \longrightarrow \mathrm{L}_{\text {alt }}^{\infty}\left(B^{n}\right) \longrightarrow \mathrm{L}_{\text {alt }}^{\infty}\left(B^{n+1}\right) \longrightarrow \ldots
$$

consisting of alternating essentially bounded cochains in $B^{n}$ is a resolution of $\mathbb{R}$ by relatively injective $G$-modules; this, Proposition 4.4 and [11] imply:

Corollary 4.5. There is a canonical isometric isomorphism

$$
\mathrm{H}_{\mathrm{cb}}^{2}(H, \mathbb{R}) \longrightarrow \mathcal{Z L}_{\mathrm{alt}}^{\infty}\left(B^{3}\right)^{H} .
$$

The right-hand side denotes the space of $H$-invariant alternating essentially bounded cocycles on $B^{3}$.

Let now $\rho: H \rightarrow G$ be a continuous homomorphism; assuming that there exists an $H$-equivariant measurable map $\varphi: B \rightarrow \check{S}$, we may define an element of $\mathcal{Z} \mathrm{L}_{\text {alt }}^{\infty}\left(B^{3}\right)^{H}$ by

$$
\left(\varphi_{3}^{*} \beta\right)\left(b_{1}, b_{2}, b_{3}\right):=\beta_{\check{S}}\left(\varphi\left(b_{1}\right), \varphi\left(b_{2}\right), \varphi\left(b_{3}\right)\right) \text {. }
$$

As a consequence of Proposition 4.3, Corollary 4.5 and [5], we have:

Proposition 4.6. Under the isomorphism

$$
\mathrm{H}_{\mathrm{cb}}^{2}(H, \mathbb{R}) \cong \mathcal{Z} \mathrm{L}_{\mathrm{alt}}^{\infty}\left(B^{3}\right)^{H}
$$

the pullback $\rho^{*}\left(\kappa_{G, \mathrm{~B}}^{\mathrm{b}}\right)$ of the class $\kappa_{G, \mathrm{~B}}^{\mathrm{b}}$ corresponds to $(1 / 2 \pi)\left(\varphi_{3}^{*} \beta\right)$.

\subsection{Boundary maps}

Under certain conditions, a boundary map as in Section 4.3 exists. We recall now a result describing general conditions implying the existence of such a specific boundary map; for a proof, see, for instance, [6].

Theorem 4.7. Let $\mathbf{G}$ be a connected semisimple algebraic group defined over $\mathbb{R}, G=$ $\mathbf{G}(\mathbb{R})^{\circ}$ and let $\mathbf{P}$ be a minimal parabolic subgroup defined over $\mathbb{R}$. Assume that the continuous homomorphism $\rho: H \rightarrow G$ has Zariski dense image and let $(B, \nu)$ be a Poisson boundary for $H$ as in Proposition 4.4. Then there exists a measurable $H$ equivariant map $\varphi: B \rightarrow G / P$. Moreover, any such map verifies that for almost every $\left(b_{1}, b_{2}\right) \in B^{2}$, the images $\varphi\left(b_{1}\right), \varphi\left(b_{2}\right)$ are transverse.

We recall that $a, b \in G / P$ are called transverse if the pair $(a, b) \in(G / P)^{2}$ belongs to the unique open $G$-orbit for the diagonal action on $G / P \times G / P$.

In particular, assuming now that the symmetric space $\mathcal{X}$ associated to $G$ is Hermitian and letting as usual $\breve{S} \subset \mathfrak{p}_{+}$denote the Shilov boundary of the bounded domain realization of $\mathcal{X}$, we have:

Corollary 4.8. Under the assumptions of Theorem 4.7, there exists a measurable equivariant map $\varphi: B \rightarrow \check{S}$ such that $\left(\varphi\left(b_{1}\right), \varphi\left(b_{2}\right)\right) \in \check{S}^{(2)}$ for almost every $\left(b_{1}, b_{2}\right) \in B^{2}$.

For the benefit of the reader we mention that an alternative condition to the Zariski density ensuring that the conclusion of Corollary 4.8 holds is that $\rho: H \rightarrow G$ is a tight homomorphism, as defined in [8]. 


\section{Proofs}

In this section we conclude the proofs of Theorems 3 and 4 and Corollaries 5 and 6 in the Introduction.

We start with a preliminary observation concerning the Hermitian triple product and keep the notation of Sections 2 and 3. In particular, we will identify the Shilov boundary $\check{S}$ with $(\mathbf{G} / \mathbf{Q})(\mathbb{R})$. Given $(a, b) \in \check{S}^{(2)}$, let $\mathcal{O}_{a, b} \subset \mathbf{G} / \mathbf{Q}$ be the Zariski open subset on which the map

$$
P_{a, b}: \mathcal{O}_{a, b} \longrightarrow \mathbb{C}^{\times} \mathbb{1} \backslash A^{\times}, \quad x \mapsto\langle\langle a, b, x\rangle\rangle_{\mathbb{C}},
$$

is defined.

Lemma 5.1. Assume that $\mathcal{X}$ is not of tube type. Then, for any $m \in \mathbb{Z}$, the map

$$
\mathcal{O}_{a, b} \longrightarrow \mathbb{C}^{\times} \mathbb{1} \backslash A^{\times}, \quad x \mapsto P_{a, b}(x)^{m},
$$

is not constant.

Proof. Observe that since $\langle\langle\cdot, \cdot, \cdot\rangle\rangle_{\mathbb{C}}$ is $G$-invariant,

$$
\langle\langle\cdot, \cdot, \cdot\rangle\rangle_{\mathbb{C}}\left(\check{S}^{(3)}\right)=P_{a, b}\left(\check{S} \cap \mathcal{O}_{a, b}\right) .
$$

If now $P_{a, b}^{m}$ were constant, then $P_{a, b}\left(\mathcal{O}_{a, b}\right)$ would be finite; but $\mathcal{O}_{a, b}$ being connected, $P_{a, b}$ would be constant, and hence $\langle\langle\cdot, \cdot, \cdot\rangle\rangle_{\mathbb{C}}$ would be constant on $\check{S}^{(3)}$; but this would imply by Corollary 2.17 that $\langle\langle\cdot, \cdot, \cdot\rangle\rangle$ is constant on $\check{S}^{(3)}$, thus contradicting Theorem 4(3).

\subsection{Proof of Theorems 3 and 4}

We prove Theorem 4 since it will imply Theorem 3 .

For $1 \leqslant i \leqslant n$, let $\rho_{i}: H \rightarrow G_{i}$ be continuous representations and set, for ease of notation, $\kappa_{i}^{\mathrm{b}}:=\kappa_{G_{i}, \mathrm{~B}}^{\mathrm{b}}$. Assume that there is a nontrivial linear relation over $\mathbb{Z}$,

$$
\sum_{i=1}^{n} m_{i} \rho_{i}^{*}\left(\kappa_{i}^{\mathrm{b}}\right)=0 .
$$

Let $(B, \nu)$ be a Poisson boundary of $H$ given by Proposition 4.4 and let $\varphi_{i}: B \rightarrow \check{S}_{i}$ be the $H$-equivariant measurable maps given by Corollary 4.8. Then (5.1), Corollary 2.17, (3.2) and Proposition 4.6 imply that, for almost every $\left(b_{1}, b_{2}, b_{3}\right) \in B^{3}$, we have

$$
\prod_{i=1}^{n}\left\langle\left\langle\varphi_{i}\left(b_{1}\right), \varphi_{i}\left(b_{2}\right), \varphi_{i}\left(b_{3}\right)\right\rangle\right\rangle_{\mathbb{C}}^{m_{i}}=1 .
$$

Consider now

$$
\rho: H \longrightarrow \prod_{i=1}^{n} \mathbf{G}_{i}, \quad h \mapsto\left(\rho_{i}(h)\right)_{i},
$$


and

$$
\varphi: B \longrightarrow \prod_{i=1}^{n} \check{S}_{i}, \quad b \mapsto\left(\varphi_{i}(b)\right)_{i},
$$

and let us define $\Lambda:=\rho(H)$ with Zariski closure $\mathbf{E}:=\bar{\Lambda}^{Z}$. Fix $\left(b_{1}, b_{2}\right) \in B^{2}$ such that, for all $1 \leqslant i \leqslant n$,

- $\left(\varphi_{i}\left(b_{1}\right), \varphi_{i}\left(b_{2}\right)\right) \in \check{S}^{(2)}$ (see Corollary 4.8); and

- (5.2) holds for almost every $b_{3} \in B$.

Set

$$
\mathcal{O}_{i}:=\mathcal{O}_{\varphi_{i}\left(b_{1}\right), \varphi_{i}\left(b_{2}\right)} \subset \mathbf{G}_{i} / \mathbf{Q}_{i}
$$

and $\mathcal{O}:=\prod_{i=1}^{n} \mathcal{O}_{i}$, and define

$$
P_{i}: \mathcal{O}_{i} \longrightarrow \mathbb{C}^{\times} \mathbb{1} \backslash A^{\times}
$$

by $P_{i}:=P_{\varphi_{i}\left(b_{1}\right), \varphi_{i}\left(b_{2}\right)}$. Then, for the essential image $\operatorname{Ess} \operatorname{Im}(\varphi)$ of $\varphi$, we have

$$
\operatorname{Ess} \operatorname{Im}(\varphi) \cap \mathcal{O} \subset\left\{\left(x_{1}, \ldots, x_{n}\right) \in \mathcal{O} \mid \prod_{i=1}^{n} P_{i}\left(x_{i}\right)^{m_{i}}=1\right\} .
$$

The right-hand side is a proper Zariski closed subset of $\mathcal{O}$ (Lemma 5.1), from which it follows that the $\mathbf{E}$-invariant subset $\overline{\operatorname{Ess} \operatorname{Im}(\varphi)}{ }^{Z} \subset \prod \mathbf{G}_{i} / \mathbf{Q}_{i}$ is proper and Zariski closed, and hence that $\mathbf{E}$ is a proper $\mathbb{R}$-subgroup of $\prod_{i=1}^{n} \mathbf{G}_{i}$. This implies, in particular, that $n \geqslant 2$ and proves that the bounded Kähler class $\rho_{i}^{*}\left(\kappa_{i}^{\mathrm{b}}\right)$ does not vanish. Since the groups $\mathbf{G}_{i}$ are all simple non-Abelian as abstract groups, and $\operatorname{pr}_{i}(\mathbf{E})=\mathbf{G}_{i}$ (recall that $\rho_{i}(H)$ is Zariski dense in $\left.\mathbf{G}_{i}\right)$, there exists a nontrivial partition $I_{1} \cup \cdots \cup I_{\ell}=\{1, \ldots, n\}$ and $\mathbb{R}$-isomorphisms $\pi_{i j}: \mathbf{G}_{i} \rightarrow \mathbf{G}_{j}$, for $i, j \in I_{k}$ for some $i \leq k \leq \ell$, such that

$$
\rho_{j}(h)=\pi_{i j} \rho_{i}(h)
$$

for all $h \in H$. In particular, there is $i \neq j$ such that $\left(\rho_{i}, \mathcal{X}_{i}\right)$ and $\left(\rho_{j}, \mathcal{X}_{j}\right)$ are equivalent, which shows Theorem 4 .

Let $n=2, m_{1}=-m_{2}=1, T_{12}: \mathcal{X}_{1} \rightarrow \mathcal{X}_{2}$ the isometry induced by $\pi_{12}$. Let $\eta=1$ if $T_{12}$ preserves the complex structure and $\eta=-1$ otherwise. Then we get

$$
\rho_{2}(h)=\pi_{12} \rho_{1}(h)
$$

for $h \in H$, and hence $(1-\eta) \rho_{1}^{*}\left(\kappa_{i}^{\mathrm{b}}\right)=0$, which - in view of the fact that $\rho_{i}^{*}\left(\kappa_{i}^{\mathrm{b}}\right) \neq 0$ implies that $\eta=1$ and $T_{12}$ is holomorphic, thus completing the proof of Theorem 3.

\subsection{Proof of Corollaries 5 and 6}

Concerning Corollary 5, we record the following proposition from [6, Prop. 9.1] which, even though concerned specifically $G=\operatorname{PSU}(p, q)$ for $1 \leqslant p<q$, only uses Theorem 4 which is valid for any $G=\operatorname{Isom}(\mathcal{X})^{\circ}$, as long as $\mathcal{X}$ is Hermitian symmetric not of tube type.

If $\Gamma$ is a finitely generated group, let $\operatorname{Rep}(\Gamma, G)$ be the quotient of $\operatorname{Hom}(\Gamma, G)$ by the $G$-conjugation and let $\operatorname{Rep}_{Z D}(\Gamma, G) \subset \operatorname{Rep}(\Gamma, G)$ be the subset consisting of representations with Zariski dense image. 
Proposition 5.2. ([6]) Assume that $G=\operatorname{Isom}(\mathcal{X})^{\circ}$ and that $\mathcal{X}$ is an irreducible Hermitian symmetric space not of tube type. Let

$$
c: I \longrightarrow \operatorname{Rep}_{Z D}(\Gamma, G)
$$

be a continuous injective path from an open interval $I \subset \mathbb{R}$, and let

$$
K: \operatorname{Rep}_{Z D}(\Gamma, G) \longrightarrow \mathrm{H}_{\mathrm{b}}^{2}(\Gamma, \mathbb{R}), \quad \rho \mapsto \rho^{*}\left(\kappa_{G, \mathrm{~B}}^{\mathrm{b}}\right) .
$$

Then

$$
\{K(c(t)) \mid t \in I\} \subset \mathrm{H}_{\mathrm{b}}^{2}(\Gamma, \mathbb{R})
$$

contains an uncountable subset which is independent over $\mathbb{R}$.

Proof of Corollary 5. If $\mathrm{H}_{\mathrm{b}}^{2}(\Gamma, \mathbb{R})$ is finite dimensional, Proposition 5.2 implies that $\operatorname{Rep}_{Z D}(\Gamma, G)$ must be zero dimensional and hence consists of finitely many points.

Proof of Corollary 6. Let $\left(\rho_{i}, \mathcal{X}_{i}\right)$ be inequivalent representations, where the $\mathcal{X}_{i}$ are irreducible Hermitian symmetric spaces not of tube type. Then

$$
\rho_{i}^{*}\left(\kappa_{G_{i}, \mathrm{~B}}\right) \in \text { Image }\left\{\mathrm{H}^{2}(\Gamma, \mathbb{Z}) \longrightarrow \mathrm{H}^{2}(\Gamma, \mathbb{R})\right\}
$$

(see Proposition 4.1). Since $\mathrm{H}_{\mathrm{b}}^{2}(\Gamma, \mathbb{R})$ injects into $\mathrm{H}^{2}(\Gamma, \mathbb{R})$, we have that the classes

$$
\left\{\rho_{i}^{*}\left(\kappa_{G_{i}, \mathrm{~B}}\right) \mid 1 \leqslant i \leqslant n\right\}
$$

are independent over $\mathbb{Z}$. Since we are dealing with ordinary cohomology and the $\rho_{i}^{*}\left(\kappa_{G_{i}, \mathrm{~B}}\right)$ satisfy $(5.3)$, they are also linearly independent over $\mathbb{R}$.

\section{References}

[1] A. Borel, Linear Algebraic Groups, 2nd ed., Graduate Texts in Mathematics, Vol. 126, Springer-Verlag, New York, 1991.

[2] M. Burger, A. Iozzi, Bounded differential forms, generalized Milnor-Wood inequality and an application to deformation rigidity, to appear in Geom. Dedicata.

[3] M. Burger, A. Iozzi, Measurable Cartan theorem and applications to deformation rigidity, preprint, 2007.

[4] M. Burger, A. Iozzi, A useful formula in bounded cohomology, to appear in Séminaires et Congres, Société Mathématique de France.

[5] M. Burger, A. Iozzi, Boundary maps in bounded cohomology, Geom. Funct. Anal. 12 (2002), 281-292.

[6] M. Burger, A. Iozzi, Bounded Kähler class rigidity of actions on Hermitian symmetric spaces, Ann. Sci. École Norm. Sup. (4) 37 (2004), no. 1, 77-103.

[7] M. Burger, A. Iozzi, A. Wienhard, Surface group representations with maximal Toledo invariant, preprint, 2006, arXiv:math.DG/0605656.

[8] M. Burger, A. Iozzi, A. Wienhard, Tight embeddings, preprint, 2007, http://www.math . ethz.ch/ iozzi/tight.pdf. 
[9] M. Burger, A. Iozzi, A. Wienhard, Surface group representations with maximal Toledo invariant, C. R. Acad. Sci. Paris, Sér. I 336 (2003), 387-390.

[10] M. Burger, N. Monod, Bounded cohomology of lattices in higher rank Lie groups, J. Eur. Math. Soc. 1 (1999), no. 2, 199-235.

[11] M. Burger, N. Monod, Continuous bounded cohomology and applications to rigidity theory, Geom. Funct. Anal. 12 (2002), 219-280.

[12] E. Cartan, Sur les groupes de la géométrie hyperspherique, Comment. Math. Helv. 4 (1932), 158-171.

[13] J. L. Clerc, A triple ratio on the unitary Stiefel manifold, Enseign. Math. (2) 48 (2002), nos. $1-2,51-71$.

[14] J. L. Clerc, An invariant for triples in the Shilov boundary of a bounded symmetric domain, preprint, 2004.

[15] J. L. Clerc, The Maslov triple index on the Shilov boundary of a classical domain, J. Geom. Phys. 49 (2004), no. 1, 21-51.

[16] J. L. Clerc, B. Ørsted, The Maslov index revisited, Transform. Groups 6 (2001), no. 4, 303-320.

[17] J. L. Clerc, B. Ørsted, The Gromov norm of the Kaehler class and the Maslov index, Asian J. Math. 7 (2003), no. 2, 269-295.

[18] A. Domic, D. Toledo, The Gromov norm of the Kaehler class of symmetric domains, Math. Ann. 276 (1987), no. 3, 425-432.

[19] J. L. Dupont, Bounds for characteristic numbers of flat bundles, in: Algebraic Topology, Aarus 1978, Lecture Notes in Mathematics, Vol. 763, Springer-Verlag, New York, 1979.

[20] J. L. Dupont, A. Guichardet, À propos de l'article: "Sur la cohomologie réelle des groupes de Lie simples réels" [Ann. Sci. Ećole Norm. Sup. (4) 11 (1978), no. 2, 277-292] par Guichardet et D. Wigner, Ann. Sci. École Norm. Sup. (4) 11 (1978), no. 2, 293-295.

[21] E. Ghys, Groupes d'homéomorphismes du cercle et cohomologie bornée, in: The Lefschetz Centennial Conference, Part III, (Mexico City, 1984), Contemp. Math., Vol. 58, American Mathematical Society, Providence, RI, 1987, pp. 81-106.

[22] W. M. Goldman, Complex Hyperbolic Geometry, Oxford Mathematical Monographs, Clarendon Press, Oxford University Press, New York, 1999.

[23] W. M. Goldman, J. Millson, Local rigidity of discrete groups acting on complex hyperbolic space, Invent. Math. 88 (1987), 495-520.

[24] A. Guichardet, D. Wigner, Sur la cohomologie réelle des groupes de Lie simples réels, Ann. Sci. École Norm. Sup. (4) 11 (1978), no. 2, 277-292.

[25] Harish-Chandra, Representations of semisimple Lie groups. VI. Integrable and squareintegrable representations, Amer. J. Math. 78 (1956), 564-628.

[26] A. Iozzi, Bounded cohomology, boundary maps, and representations into $\mathrm{Homeo}_{+}\left(S^{1}\right)$ and $\mathrm{SU}(1, n)$, in: Rigidity in Dynamics and Geometry, Cambridge, UK, 2000, Springer-Verlag, New York, 2002, pp. 237-260.

[27] V. A. Kaimanovich, Double ergodicity of the Poisson boundary and applications to bounded cohomology, Geom. Funct. Anal. 13 (2003), no. 4, 852-861.

[28] A. Korányi, Function spaces on bounded symmetric domains, in: Analysis and Geometry on Complex Homogeneous Domains (J. Faraut, S. Kaneyuki, A. Korányi, Qi-keng Lu, G. Roos, eds.), Vol. 185, Birkhäuser, Boston, 2000, pp. 183-281. 
[29] A. Korányi, J. A. Wolf, Realization of hermitian symmetric spaces as generalized halfplanes, Ann. of Math. (2) 81 (1965), 265-288.

[30] V. Koziarz, J. Maubon, Harmonic maps and representations of non-uniform lattices of $\mathrm{PU}(m, 1)$, preprint, 2003, arXiv:math.DG/0309193.

[31] S. Matsumoto, Some remarks on foliated $S^{1}$ bundles, Invent. Math. 90 (1987), 343-358.

[32] N. Monod, Continuous Bounded Cohomology of Locally Compact Groups, Lecture Notes in Mathematics, Vol. 1758, Springer-Verlag, Berlin, 2001.

[33] C. C. Moore, Group extensions and cohomology for locally compact groups. III, Trans. Amer. Math. Soc. 221 (1976), no. 1, 1-33.

[34] I. Satake, Algebraic Structures of Symmetric Domains, Kanô Memorial Lectures, Vol. 4, Iwanami Shoten, Tokyo, 1980.

[35] D. Toledo, Representations of surface groups in complex hyperbolic space, J. Differential Geom. 29 (1989), no. 1, 125-133. 